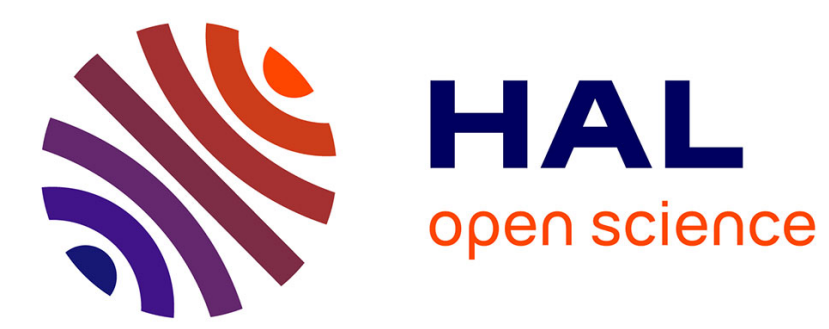

\title{
Multi-player, Multi-prize, Imperfectly Discriminating Contests
}

\author{
André de Palma, Soumyanetra Munshi
}

\section{To cite this version:}

André de Palma, Soumyanetra Munshi. Multi-player, Multi-prize, Imperfectly Discriminating Contests. 2012. hal-00683688

\section{HAL Id: hal-00683688 \\ https://hal.science/hal-00683688}

Preprint submitted on 29 Mar 2012

HAL is a multi-disciplinary open access archive for the deposit and dissemination of scientific research documents, whether they are published or not. The documents may come from teaching and research institutions in France or abroad, or from public or private research centers.
L'archive ouverte pluridisciplinaire HAL, est destinée au dépôt et à la diffusion de documents scientifiques de niveau recherche, publiés ou non, émanant des établissements d'enseignement et de recherche français ou étrangers, des laboratoires publics ou privés. 


\section{Title Page}

Title:

Multi-player, Multi-prize, Imperfectly Discriminating Contests

\section{Authors:}

(1) Name: André de Palma

Affiliation(s): Ecole Normale Supérieure de Cachan, and CES (Centre d'économie de la Sorbonne)

Address: 61, Avenue du Président Wilson, 94235 Cachan, Cedex France. Telephone: 33663644320 .

Fax: 33147402460

Email id: andre.depalma@ens-cachan.fr

(2) Name: Soumyanetra Munshi (corresponding author)

Affiliation(s): Indian Institute of Management Bangalore (IIMB)

Address: Faculty Room \# E 008, IIMB, Bannerghatta Road, Bangalore 560076, Karnataka, India.

Telephone: +91 8026993198.

Fax: +91 8026584050 .

Email id: soumyanetra.munshi@iimb.ernet.in 


\title{
MULTI-PLAYER, MULTI-PRIZE, IMPERFECTLY DISCRIMINATING CONTESTS
}

\author{
ANDRÉ DE PALMA AND SOUMYANETRA MUNSHI
}

\begin{abstract}
This paper models success probability in imperfectly discriminating contests involving multiple players and multiple prizes. This turns out to be a generalization of Tullock's contest success function to a multiplayer, multi-prize situation. The model can be used to analyse efforts exerted by individuals in various real-life situations, like obtaining seats in congested public transportation vehicles or obtaining admission into elite educational institutes. We propose a "holistic" probability model, derive the equilibrium efforts exerted, and analyse those efforts, the associated total costs and total dissipation, and also pricing and the optimal number of 'seats'. We also discuss extensions of the model.
\end{abstract}

JEL Codes: C72, D04, R41

Acknowledgement. The first author would like to thank the seminar participants at ETHZ (Civil Engineering), Cambridge University, Catholic University of Leuven (Economics), University of Copenhagen (Economics), University of Louvain-La-Neuve, Ecole Normale Supérieure de Cachan and Ecole Polytechnique, France, as well as Simon Anderson (University of Virginia), Mogens Fosgerau (Technical university of Copenhagen), Robin Lindsey, (University of British Colombia) and Jean-Luc Prigent (University of Cergy-Pontoise). He would also like to thank the following organizations: Tarification des transports individuels et collectifs à Paris. Dynamique de l'acceptabilité: Predit and Ademe. Surprice project, Scheduling, trip timing and scheduling preferences, Predit. He would also like to thank his former student Sue Wang (MIT) and and Charles Maurin (Columbia). The second author would like to acknowledge the hospitality of the faculty and students of Ecole Normale Supérieure de Cachan - this project was initiated during her visit there. She would also like to thank Prof. Barry Sopher of Rutgers, the state University of New Jersey, for his academic as well as non-academic help, and Professors Kalyan Chatterjee of Pennsylvania State University, Joan Walker of University of California Berkeley, and Krishnendu Ghosh Dastidar of Jawaharlal Nehru University, for fruitful discussions about the paper. Last, but not the least, she would like to thank Prof. Tomas Sjöström of Rutgers University for his versatile and helpful advice. 


\section{INTRODUCTION}

A contest is a competitive situation in which players expend resources to win a prize. The players could be individuals, groups, firms, lobbies, and even countries, while the resources spent could be effort, and various kinds of expenditures like expenditure on political campaigns, R \& D development, or those on arms and ammunitions build-up. Contests can be of two types - perfectly discriminating or imperfectly discriminating (Hillman and Riley [12]). In a perfectly discriminating contest, the players devoting the highest resources for the prize, are the winners. An example of such a contest would be an all-pay auction (see Clark and Riis [6], Moldovanu and Sela [19], for example).

This paper is about imperfectly discriminating contests. In such a contest, the players devoting the highest resources have the highest probability of winning but do not necessarily win the contest with certainty. Examples in this category would include political rent-seeking (Nitzan [21]), Dixit [9]), wars and battles between countries (Hwang [14]), research development and patent races (Nti $[22]$ ), promotion of workers (Rosen [25]), and competition in sports (Szymanski $[28])$.

In such imperfectly discriminating contests, the technology (function) that translates a player's expended resources, into his probability of winning is called the contest success function (CSF). Most of the studies of such contests use the logit or ratio-form CSF introduced by Tullock (Tullock [29]) ${ }^{1}$. Hirshleifer (Hirshleifer [13]) had used the difference-form CSF ${ }^{2}$. Skaperdas [26] and Clark and Riis [7] have axiomatized Tullock's CSF. Many extensions of this CSF have also been studied. These include, for example, axiomatization

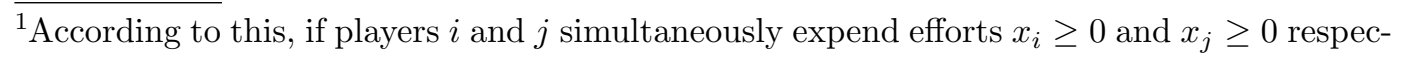
tively, then the probability of player $i$ winning the contest is described by (see Chowdhury and Sheremeta [8], Hwang [14], for example):

$$
p_{i}\left(x_{i}, x_{j}\right)=\left\{\begin{array}{l}
\frac{x_{i}}{x_{i}+x_{j}}, \text { if } x_{i}+x_{j} \neq 0 \\
\frac{1}{2}, \text { if } x_{i}=x_{j}=0 .
\end{array}\right.
$$

${ }^{2}$ According to this, if players $i$ and $j$ simultaneously expend efforts $x_{i} \geq 0$ and $x_{j} \geq 0$ respectively, then the probability of player $i$ winning the contest is described by (see Hwang [14], for example):

$$
p_{i}\left(x_{i}, x_{j}\right)=\frac{\exp \left(x_{i}\right)}{\exp \left(x_{i}\right)+\exp \left(x_{j}\right)}
$$


of the CSF with the possibility of a draw (Blavatskyy [4]), integration of the difference and the ratio-forms axiomatically and stochastically (Hwang [14]), extension of axiomatization of the ratio-form CSF to include groups of players (Münster [20]), among others.

In all these studies, the focus has been on a single prize. For example, the rent-seeking literature dwells mostly on winning a single prespecified rent. The few extensions in multi-rent situations (like Clark and Riis [6] and Gradstein and Nitzan [11]) the contest outcome functions are assumed to be perfectly discriminating - so that the setting is essentially one of a generalized first-price all-pay auction (Nitzan [21] and Clark and Riis [6]). However, many real-life imperfectly discriminating contests can involve multiple prizes. (Consider the examples below.)

Essentially, the attempt here is to model a situation where a limited number of identical prizes are unpriced, and so only individual effort can increase the chance of a player gaining access to it. In doing so, however, the standard approach starts with individual preferences, and then goes on to consider aggregation (sometimes even taking into account capacity constraints, as is necessary in our case, where the number of prizes is smaller than the number of players competing for them $)^{3}$.

Here, on the contrary, we propose some plausible assumptions on outcomes at the aggregate level, and then derive individual behavior from it ${ }^{4}$. This approach is thus radically different, and to differentiate we call it a "holistic" approach (as opposed to the usual individualistic approach), which refer to the approach of beginning with the group behavior and then deriving individual behavior from it.

\footnotetext{
${ }^{3}$ For example, de Palma, Picard and Waddell [3], consider an axiomatic approach to deal with the excess demand (and the queue discipline).

${ }^{4}$ The approach is similar in spirit to the generic principal-agent framework: In this case, the agent exerts some effort which is non-observable to the principal, though the resultant output of such effort (say, production) is observed. The output, moreover, is typically probabilistically related to effort exerted. Hence the principal, who wishes to maximize effort, designs optimal contracts to that end (see Laffont and Martimort [15]). In our model, similarly, individual efforts are not directly observed. However the outcome (the fact that a passenger has access to a seat) is observable. As such, we can derive the individual effort level from the resulting aggregate outcome (where some of the passengers get to sit). Moreover, as expected, individual effort depends on efforts of all the competing individuals (hence our set-up is one of an 'aggregate game' where a player's payoff depends on his own effort and on the aggregate efforts of all the players, see Martimort and Stole [19]).
} 
In Section II, the basic model that relates individual efforts to aggregate outcomes, is described (we call the fundamental premise of this model, the "Holistic" Approach with Single Effort). In an extension of the basic model (see Section IV), we consider a situation where the individual can exert more than one level of effort and employ a similar approach to study individual level variables of interest. (We call this approach the "Holistic" Approach with Dual Efforts.)

In the "Holistic" Approach with Single Effort model, efforts of all the individuals translate into the aggregate probability of a possible outcome, like some passengers sitting in a limited number of seats in a public transport vehicle (while others remain standing), or some students getting admitted in institutes of higher education (while others not being accepted), or some households getting allotted houses in a regulated housing market (while not others). From the model, we derive the probability of an individual being successful (getting to sit, getting admission to a coveted Institute, etc.). This probability reduces to Tullock's CSF in case of a single prize. Hence the scope of this model is to better understand such imperfectly discriminating contests with multiple prizes and multiple players. The formulation turns out to be a generalization of Tullock's CSF.

We then use this probability framework to study optimal effort exertion in specific contexts. For example, in our setting, the high quality good (prize) is subject to scarcity, while the low quality good (which a player will consume by default if he fails to get the prize) is subject to congestion (meaning an increase in the number of individuals consuming the low quality good, will further lower its quality, or in other terms, increase the cost of consuming it). However, it is always preferable to get the higher quality good, even if the lower quality good is not subject to congestion. Moreover, a player may or may not obtain a unit of the prize, while no player can be excluded from the low quality good.

Our analysis yields that when costs of effort are symmetric among players, then a symmetric Nash equilibrium exists in which all players exert the same level of effort, and this effort first increases and then falls with congestion. We also compute comparative statics of other variables of interest like total cost and total dissipation. In this model we also analyse the optimal number of prizes as a matter of policy instrument for the public authorities. 
We have also envisaged an extension of the basic model in this paper (Section IV). Here we study effort exertion with two different types of goods, a better quality and a lower quality good. If an agent does not get the high or the low quality good, he has access to a third alternative (worse than getting any of the two goods). Beginning with the "holistic" approach, and then deriving individual probabilities of success, we show that in (the symmetric) equilibrium, all effort will be devoted to having access to the better quality alternative.

As an analysis of scarce resource facing excessive demand, the proposed approach is amenable to different contexts as mentioned before. Below we list the essential characteristics of some such markets that make our model particularly applicable.

Contest for seats in public transportation: Overwhelming demand for public transport is a reality in many urban centres, especially in emerging economies like India. Trains and buses, plying in and out of the cities in India, are dangerously overcrowded ${ }^{5}$. Situation on buses in Indian cities is similar. Buses are severely overcrowded, with some passengers forced to ride on the outsides of vehicles and even on the roofs.

First note that once inside the bus/train, there are many (similar) seats, hence this is a multi-prize contest with similar prizes. Moreover passengers will exert effort to get seated, but there is no guarantee that the people exerting highest efforts will secure seats for themselves - they will merely increase the probability of getting seated. Hence the contest for seats is imperfectly discriminating. It is likely that in this case, the effort exerted by an individual to get a seat increases the more there are people competing for it.

In fact, here is an additional complexity here - the more are the people that remain standing, the greater will be the discomfort in standing, due to limited available space. As a consequence, it is possible that the effort exerted to get a seat increases as the total number of users increases (since standing becomes

\footnotetext{
${ }^{5}$ For example, on suburban rail lines in Mumbai (that carry passengers from the suburbs of Mumbai into Mumbai), peak-hour trains carry more than twice their maximum capacity, leading to inhuman travelling conditions, with so-called 'super dense crush loads' of 14 to 16 standing passengers per square meter of floor space. On peak-hour trains, many passengers are forced to hang out doors and windows or to ride between train cars or even hang on the outsides of cars (see Pucher, Korattyswaroopam, and Ittyerah [24]).
} 
even more worse). This means that there is a spillover effect since the person who is ultimately getting to sit is also having a higher level of cost of travel (which includes cost of exerting effort) when congestion is higher since he is having to exert much greater effort to get a seat ${ }^{6}$.

Different aspects of the transportation problem have extensively been studied in the transportation literature and to some extent in the economics literature ${ }^{7}$. Vickrey [31] discusses a model to study congestion of a road at a single bottleneck during peak time. Arnott, de Palma and Lindsey [2] study a structural model, as an extension of Vickrey's model ([31]) with a bottleneck congestion for private transportation. Users compete to have access to a limited capacity (a bottleneck, access to a bridge or a tunnel). The solution is to bet for the better time slice. Hence, like in our present model, when queues are stochastic, the situation resembles an imperfectly discriminating contest.

However our approach is different from these studies in that we exclusively concentrate within a specific transportation vehicle and explicitly model efforts of individuals and relate them probabilistically to success (defined as obtaining a place to sit). In one of the extensions (see Section IV), we model two kinds of efforts which, in the context of transportation, can be thought of as boarding and sitting efforts.

Contest for admission into elite educational institutions: Admission into relatively very few seats in elite educational institutions is again, an imperfectly discriminating contest with multiple symmetric prizes - For instance, in several countries, there is a numerous clausus. Consider an education system, as in France, with the Grandes Écoles (which in French literally mean "great schools" or "elite schools") being higher education establishments outside the main framework of the French university system. Universities mainly allow students to organize their work the way they wish, while the work activities are much more organized in Grandes Écoles (with a feeling of lost of freedom for some students).

\footnotetext{
${ }^{6}$ This situation may not be representative of situations in many developed countries like Sweden, for example, where, if a bus is crowded, in case of a vacant seat, people politely stand and wait for someone else to sit down.

${ }^{7}$ Mohring [18] discusses the problems of urban public transport - more generally the effect of a decline in demand for a product that involves increasing returns to scale. Vickrey [30] discusses pricing issues with urban and suburban transport while Parry and Small [23] studies the same for urban transport.
} 
Unlike French public universities which have an obligation to accept all candidates of the same region who hold a bachelor's degree (baccalauréat), the selection criteria of Grandes Écoles rest mainly on competitive written and oral exams, usually undertaken by students from dedicated preparatory classes. Some students prefer the university, where basically everyone with a baccalauréat is accepted since there are no capacity constraint in the university, but, the level and/or the prestige of the Grandes Écoles is higher. Many students therefore, aspire to go to Grandes Écoles, but all are not able to do so due to limited intake of students 8 . And chances are probably higher when a candidate has prepared and studied hard. (In such a case, however, effort should be encouraged, since it is not a dissipation and the effort devoted by a student is not necessarily a social loss, unlike the case of public transportation, for example.)

Contest for getting housing in regulated housing market: A possible third example is provided by the housing market in France. Usually two systems exist - one is an unregulated market where a market clearing price prevails given a free market and the other, a regulated market. In the latter case, there are long queues to have access to the housing units, but with good enough arguments, appropriate letters, some phone calls to the right person and at the right time, in order words, with a sufficient level of effort, one can get a housing unit in the regulated market. Hence competition for housing in the regulated market is a situation of imperfectly discriminating multi-prize contest.

Contest for entering 'pandals': In India, during festivities, large make-shift temporary structures of cloth and bamboo are created and decorated (often called pandals, in local language). These pandals house Indian gods and goddesses, and devotees and revellers make a beeline to catch a glimpse of magnificent art and decoration. Entry is free but such pandals are so crowded that people have to make sufficient efforts (stand in queues for long hours, walk long distances etc.) to be able to make it inside - those making more effort are more likely to succeed in catching a glimpse of all the artwork inside. However to avoid queues, lot of other avenues are often explored - bribes are handsomely paid to organizing committees to get entry from other sides, they try and get VIP passes so that they can bypass the queue. All such actions can be referred to as "effort".

${ }^{8}$ They do not have a large student body - about 3,000 students at the largest establishment most have a few hundred students each year. 
Contest for getting goods in church 'give-aways': Often churches in the US organize give-away's, where furniture and other household objects (like lamps, mattresses, tables, chairs, utensils etc.) that have been donated by households to a church, are put up in public for graduate students (and other interested persons) to take away. All graduate students assemble ahead of time, and when the right time is signalled, all students make a dash to get objects they desire. Often many students may want the same thing, in which case, whoever goes and touches the object first, gets it. Hence, like before, it is a situation where higher effort is likely to increase the probability of success (of getting the desired object).

Similarly, we could think of other examples where our model could be applied like competition among cars for parking space adjacent to theatres, shopping malls, competition for a higher rank/position in a large organization, or competition to have access to medical care in highly congested medical systems.

The rest of the paper is organized as follows: Section II ( Holistic" Approach with Single Effort) lays down the model relating efforts to outcomes. The next Section lays down the equilibrium analysis and comparative statics. Section IV ("Holistic" Approach with Dual Efforts) extends the model by including two kinds of efforts while Section $\mathrm{V}$ analyses the model with heterogeneous populations. Section VI concludes.

\section{The Model}

II(i). Probability Model: Say there are $n$ players/travellers and $\bar{n}<n$, many prizes/seats (which correspond to seats in a bus/train in the transportation example, or seats in a coveted educational Institute, for example). (We cast our model with the transportation example in mind for easy exposition, but of course, it can be applied to several other situations, as explained in the introduction.)

The set of all possible outcomes can be represented by the collection of vectors

$$
\Omega:=\left\{\mathbf{v}=\left(\varepsilon_{1}(\mathbf{v}), \ldots, \varepsilon_{n}(\mathbf{v})\right) \in\{0,1\}^{n}: \sum_{i=1}^{n} \varepsilon_{i}(\mathbf{v})=\bar{n}\right\} .
$$

The restriction $\sum_{i=1}^{n} \varepsilon_{i}(\mathbf{v})=\bar{n}$ reflects the fact that in any outcome all the seats are occupied (in the bus or in the educational Institute), that is, all 
prizes are taken. Note that here $\varepsilon_{i}(\mathbf{v})=1$ means that in the outcome $\mathbf{v}$ the $i$-th person is successful (in sitting or getting admitted), and otherwise $\varepsilon_{i}(\mathbf{v})=0$. Also note that the number of different outcomes in the space is given by

$$
|\Omega|=\left(\begin{array}{l}
n \\
\bar{n}
\end{array}\right)=\frac{n !}{\bar{n} !(n-\bar{n}) !} .
$$

A probability on the space $\Omega$ is given by a collection $P$ of non-negative real numbers $p_{\mathbf{v}}$ allotted to each outcomes $\mathbf{v} \in \Omega$, satisfying the two conditions

$$
p_{\mathbf{v}} \geq 0, \quad \text { and } \quad \sum_{\mathbf{v} \in \Omega} p_{\mathbf{v}}=1 .
$$

The pair $(\Omega, P)$ is said to form a probability space.

The question we are asking is the following: Can we have a model (a probability space, of course the sample space $\Omega$ should be the one given above) such that the probability of any outcome reflects the collective effort of the mode of the outcome (the way the outcome comes about)? That is we introduce a new assumption that describes the likely outcome (realization) of the system. It is reminiscent of the approach used in statistical mechanics, however our approach is different and new to the best of our knowledge. Here, from the aggregate assumption we are able to recover individual probabilities.

Assumption 1. "Holistic" Approach with Single Effort: Let probability $p_{\mathbf{v}}$ be proportional to the sum of the efforts of the agents who are successful in the outcome $\mathbf{v}$ as well as the efforts of the agents who are exerting efforts to be successful but are unable to do so. That is,

$$
p_{\mathbf{v}} \propto\left[\sum_{i=1}^{n} \varepsilon_{i}(\mathbf{v}) e_{i}+\lambda \sum_{i=1}^{n}\left(1-\varepsilon_{i}(\mathbf{v})\right) e_{i}\right]
$$

where $e_{i}$ be the effort exerted by the ith agent to obtain a unit of the better quality good out of $\bar{n}$ available units, with $\bar{n} \leq n, i=1, \ldots, n$, and $0 \leq \lambda \leq 1$.

This assumption means that there exists a positive constant (not depending on the outcome) $K$ such that

$$
p_{\mathbf{v}}=K\left[\sum_{i=1}^{n} \varepsilon_{i}(\mathbf{v}) e_{i}+\lambda \sum_{i=1}^{n}\left(1-\varepsilon_{i}(\mathbf{v})\right) e_{i}\right] .
$$


The following proposition lays down the value of $K$, the probability of an outcome, $p_{\mathbf{v}}$, and the probability of 'success' for player $i, \operatorname{Pr}\left\{\varepsilon_{i}=1\right\}$ :

Proposition 1. Consider the probability space given by (1). Then, under Assumption (1), the probability of outcome $\mathbf{v}$ is given by

$$
p_{\mathbf{v}}=K\left[\sum_{i=1}^{n} \varepsilon_{i}(\mathbf{v}) e_{i}+\lambda \sum_{i=1}^{n}\left(1-\varepsilon_{i}(\mathbf{v})\right) e_{i}\right]
$$

where the constant of proportionality $K$ is given by

$$
K=\frac{1}{\frac{(n-1) !}{(\bar{n}-1) !(n-\bar{n}) !}\left(\frac{\bar{n}+\lambda(n-\bar{n})}{\bar{n}}\right) \sum_{i=1}^{n} e_{i}} .
$$

Moreover, the probability of success for player $i$ is:

$$
\operatorname{Pr}\left\{\varepsilon_{i}=1\right\}=\frac{\bar{n}(\bar{n}-1+\lambda(n-\bar{n}))}{(n-1)(\bar{n}+\lambda(n-\bar{n}))}+\frac{e_{i}}{\sum_{j} e_{j}} \frac{(n-\bar{n})}{(n-1)} \frac{\bar{n}(1-\lambda)}{(\bar{n}+\lambda(n-\bar{n}))} .
$$

Proof. Derivation of $K$ : The derivation of the constant of proportionality $K$ is as follows: The non-negativity of $p_{\mathbf{v}}$ just means that $K$ has to be nonnegative (as the efforts $e_{i} \geq 0$ ). The second criterion is satisfied if and only if

$$
1=\sum_{\mathbf{v} \in \Omega} p_{\mathbf{v}}=\sum_{\mathbf{v} \in \Omega}\left[K\left[\sum_{i=1}^{n} \varepsilon_{i}(\mathbf{v}) e_{i}+\lambda \sum_{i=1}^{n}\left(1-\varepsilon_{i}(\mathbf{v})\right) e_{i}\right]\right] .
$$

Since $K$ is an absolute constant and not depending $i$ or $\mathbf{v}$ we can take it out of the summations to get

$$
1=K \sum_{\mathbf{v} \in \Omega}\left[\sum_{i=1}^{n} \varepsilon_{i}(\mathbf{v}) e_{i}+\lambda \sum_{i=1}^{n}\left(1-\varepsilon_{i}(\mathbf{v})\right) e_{i}\right] .
$$

Next we interchange the order of summations

$$
1=K\left\{\left[\sum_{i=1}^{n} \sum_{\mathbf{v} \in \Omega} \varepsilon_{i}(\mathbf{v}) e_{i}\right]+\left[\lambda \sum_{i=1}^{n} \sum_{\mathbf{v} \in \Omega}\left(1-\varepsilon_{i}(\mathbf{v})\right) e_{i}\right]\right\} .
$$

In the inner sum since the effort $e_{i}$ does not depend on $\mathbf{v}$ (this is by assumption, since we do not allow different efforts for different outcomes) we can take it out to get

$$
1=K\left\{\sum_{i=1}^{n} e_{i}\left[\sum_{\mathbf{v} \in \Omega} \varepsilon_{i}(\mathbf{v})\right]+\lambda \sum_{i=1}^{n} e_{i}\left[\sum_{\mathbf{v} \in \Omega}\left(1-\varepsilon_{i}(\mathbf{v})\right)\right]\right\} .
$$


Now consider the inner sums over $\mathbf{v}$. Here $\sum_{\mathbf{v} \in \Omega} \varepsilon_{i}(\mathbf{v})$ is just the count for the number of outcomes $\mathbf{v} \in \Omega$ for which $\varepsilon_{i}(\mathbf{v})=1$. That is,

$$
\sum_{\mathbf{v} \in \Omega} \varepsilon_{i}(\mathbf{v})=\sum_{\substack{\mathbf{v} \in \Omega \\ \varepsilon_{i}(\mathbf{v})=1}} 1
$$

From simple combinatorics we get that this count is same as the number of vectors of length $n-1$, with 0,1 entries such that $\bar{n}-1$ entries have value 1 and others have value 0 . This is given by $\left(\begin{array}{c}n-1 \\ \bar{n}-1\end{array}\right)$. Similarly, $\sum_{\mathbf{v} \in \Omega}\left(1-\varepsilon_{i}(\mathbf{v})\right)$ is just the count for the number of outcomes $\mathbf{v} \in \Omega$ for which $\varepsilon_{i}(\mathbf{v})=0$ (since otherwise the contribution is 0 ). That is,

$$
\sum_{\mathbf{v} \in \Omega}\left(1-\varepsilon_{i}(\mathbf{v})\right)=\sum_{\substack{\mathbf{v} \in \Omega \\ \varepsilon_{i}(\mathbf{v})=0}} 1 .
$$

From simple combinatorics we get that this count is same as the number of vectors of length $n-1$, with 0,1 entries such that $\bar{n}$ entries have value 1 and others have value 0 . This is given by $\left(\begin{array}{c}n-1 \\ \bar{n}\end{array}\right)$. Substituting we get the following calculations

$$
\begin{aligned}
1 & =K\left\{\sum_{i=1}^{n} e_{i}\left(\begin{array}{c}
n-1 \\
\bar{n}-1
\end{array}\right)+\lambda \sum_{i=1}^{n} e_{i}\left(\begin{array}{c}
n-1 \\
\bar{n}
\end{array}\right)\right\} \\
& =K\left(\sum_{i=1}^{n} e_{i}\right) \frac{(n-1) !}{(\bar{n}-1) !(n-\bar{n}) !}\left[\frac{1}{n-\bar{n}}+\frac{\lambda}{\bar{n}}\right] .
\end{aligned}
$$

Hence

$$
K=\frac{1}{\frac{(n-1) !}{(\bar{n}-1) !(n-\bar{n}) !}\left(\frac{\bar{n}+\lambda(n-\bar{n})}{\bar{n}}\right) \sum_{i=1}^{n} e_{i}} .
$$

Substituting we can get $p_{\mathbf{v}}$. This completes the derivation of the probability space in the case of our model. Now we come to the next part of the proposition.

Derivation of individual's probability of success: Consider person 1 . Let us compute the probability that person 1 is successful using the above probability model:

$$
\begin{aligned}
\operatorname{Pr}\left\{\varepsilon_{1}=1\right\} & =\sum_{\substack{\mathbf{v} \in \Omega \\
\varepsilon_{1}(\mathbf{v})=1}} p_{\mathbf{v}} \\
& =\sum_{\substack{\mathbf{v} \in \Omega \\
\varepsilon_{1}(\mathbf{v})=1}} K\left[\sum_{i=1}^{n} \varepsilon_{i}(\mathbf{v}) e_{i}+\lambda \sum_{i=1}^{n}\left(1-\varepsilon_{i}(\mathbf{v})\right) e_{i}\right]
\end{aligned}
$$


where $K$ is as given by (4)

$$
\begin{aligned}
& =K\left[\sum_{i=1}^{n} e_{i} \sum_{\substack{\mathbf{v} \in \Omega \\
\varepsilon_{1}(\mathbf{v})=1}} \varepsilon_{i}(\mathbf{v})+\lambda \sum_{i=1}^{n} e_{i} \sum_{\substack{\mathbf{v} \in \Omega \\
\varepsilon_{1}(\mathbf{v})=1}}\left(1-\varepsilon_{i}(\mathbf{v})\right)\right] \\
& =K\left[\sum_{i=1}^{n} e_{i} \sum_{\substack{\mathbf{v} \in \Omega \\
\varepsilon_{1}(\mathbf{v})=1 \\
\varepsilon_{i}(\mathbf{v})=1}} 1+\lambda \sum_{i=1}^{n} e_{i} \sum_{\substack{\mathbf{v} \in \Omega \\
\varepsilon_{1}(\mathbf{v})=1 \\
\varepsilon_{i}(\mathbf{v})=0}} 1\right] .
\end{aligned}
$$

Now

$$
\sum_{\substack{\mathbf{v} \in \Omega \\
\varepsilon_{1}(\mathbf{v})=1 \\
\varepsilon_{i}(\mathbf{v})=1}} 1=\left\{\begin{array}{lll}
\left(\begin{array}{l}
n-1 \\
\bar{n}-1
\end{array}\right) & \text { if } & i=1 \\
\left(\begin{array}{l}
n-2 \\
\bar{n}-2
\end{array}\right) & \text { if } & i \neq 1
\end{array},\right.
$$

and

$$
\sum_{\substack{\mathbf{v} \in \Omega \\
\varepsilon_{1}(\mathbf{v})=1 \\
\varepsilon_{i}(\mathbf{v})=0}} 1=\left\{\begin{array}{lll}
0 & \text { if } & i=1 \\
\left(\begin{array}{l}
n-2 \\
\bar{n}-1
\end{array}\right) & \text { if } & i \neq 1
\end{array} .\right.
$$

Substituting and simplifying, we get

$$
\operatorname{Pr}\left\{\varepsilon_{1}=1\right\}=\frac{\bar{n}(\bar{n}-1+\lambda(n-\bar{n}))}{(n-1)(\bar{n}+\lambda(n-\bar{n}))}+\frac{e_{1}}{\sum_{i} e_{i}} \frac{(n-\bar{n})}{(n-1)} \frac{\bar{n}(1-\lambda)}{(\bar{n}+\lambda(n-\bar{n}))} .
$$

Substituting subscript 1 with $i$, we can get (5). This completes the proof.

Notice that in this model if $\lambda=0$, we see that the probability of any outcome depends on the efforts of the people who are successful only, while $\lambda=1$ means the probability of any outcome depends equally on the efforts of those who are successful as well as those who are not so. For any $0<\lambda<1$, it means the efforts of those who are successful contribute more towards bringing about the outcome than the efforts of those who remain unsuccessful.

II(ii). Heuristic interpretation of probability. Consider the transportation example for an interpretation of the probability as follows: Let $n$ people be divided into $\phi$ number of clusters, with $\frac{n}{\phi}$ number of people in each cluster, and let each cluster fight for $\frac{\bar{n}}{\phi}$ seats. For example, let $n=100, \bar{n}=50$, and $\phi=25$. This means that there are 100 people divided into 25 groups of 4 people each, and each group is fighting for 2 seats. 
More generally, consider individual $i$ belonging to cluster $J$. In this case,

$\operatorname{Pr}\left\{\varepsilon_{i}=1\right\}=\frac{\bar{n}}{(n-\phi)(\bar{n}+\lambda(n-\bar{n}))}\left[\bar{n}-\phi+\lambda(n-\bar{n})+(n-\bar{n})(1-\lambda) \frac{e_{i}}{\sum_{j \in J} e_{j}}\right]$

which we get by replacing $n$ by $\frac{n}{\phi}$, and $\bar{n}$ by $\frac{\bar{n}}{\phi}$, and by summing efforts of people in the cluster in which $i$ belongs. Now as $\phi \rightarrow \bar{n}$, we get

$$
\operatorname{Pr}\left\{\varepsilon_{i}=1\right\}=\frac{\bar{n}}{\bar{n}+\lambda(n-\bar{n})}\left[\lambda+(1-\lambda) \frac{e_{i}}{\sum_{j \in J} e_{j}}\right] .
$$

Moreover, if now $\lambda \rightarrow 0$, we get the Logit functional form $\operatorname{as}^{9}$ :

$$
\operatorname{Pr}\left\{\varepsilon_{i}=1\right\}=\frac{e_{i}}{\sum_{j \in J} e_{j}} .
$$

That is, when $n$ people are divided into $\bar{n}$ groups of $\frac{n}{\bar{n}}$ people each, so that each group is fighting for 1 seat, moreover the weight attached to the efforts of people who cannot sit is 0 , then the probability of getting to sit is just the relative effort.

Now suppose the sum of efforts in each cluster was equal, then $\sum_{j \in J} e_{j}=$ $\sum_{i} e_{i} / \bar{n}$. Hence, in this case, we get

$$
\operatorname{Pr}\left\{\varepsilon_{i}=1\right\}=\bar{n} \frac{e_{i}}{\sum_{j} e_{j}} .
$$

We can verify that we get $\sum_{i} \operatorname{Pr}\left\{\varepsilon_{i}=1\right\}=\bar{n}$ (see Observation 3 below for details).

II(iii). Properties of the probability model: Other observations that follow from the general formulation are enumerated as follows:

${ }^{9}$ Also notice that the probability to get the better quality good can be interpreted as in a dogit model. The dogit model is written as:

$$
p_{i}=\frac{e^{V_{i}}+\theta_{i} \sum_{j} e^{V_{j}}}{\left(1+\sum_{j} \theta_{j}\right) \sum_{j} e^{V_{j}}} ; i, j=1, \ldots, N,
$$

where $p_{i}$ is the probability of choosing $i$ th of the $N$ alternatives, $V_{i}$ is a function describing attributes of the $i$ th alternative, and $\theta_{i}$ is a non-negative parameter associated with the $i$ th alternative. Notice that this probability can be re-written as

$$
p_{i}=\frac{\theta_{i}}{1+\sum_{j} \theta_{j}}+\frac{1}{\left(1+\sum_{j} \theta_{j}\right)} \frac{e^{V_{i}}}{\sum_{j} e^{V_{j}}} ; i, j=1, \ldots, N
$$

which is of the same form (with $V_{i}$ 's set to 1 ) as the probability of getting the better quality good in (5). See Anderson, de Palma, and Thisse [1], and Gaudry and Dagenais [10], for example. 
Observation 1. If $\lambda=1$, we get

$$
\operatorname{Pr}\left\{\varepsilon_{i}=1\right\}=\frac{\bar{n}}{n}
$$

which is the equal probability model. This means that if people's efforts are equally weighted in the probability of an outcome, irrespective of whether they are ultimately successful or not, then each individual has the same probability of being successful, irrespective of effort levels.

Observation 2. If effort is symmetric, that is $e_{i}=e$, we get

$$
\operatorname{Pr}\left\{\varepsilon_{i}=1\right\}=\frac{\bar{n}}{n}, \forall i
$$

again yielding the equal probability model. This means that if every individual exerts the same effort, then each person's probability of being successful is the same, irrespective of the weight of the efforts of the people who are ultimately not successful $(\lambda)$.

As expected, we can check that for an individual exerting maximum effort, that is for $e_{i}=\max _{j}\left\{e_{j}\right\}$, we have $\frac{e_{i}}{\sum_{j} e_{j}}>\frac{1}{n}$, so that $\operatorname{Pr}\left\{\varepsilon_{i}=1\right\}>\frac{\bar{n}}{n}$. Similarly, for an individual exerting minimum effort, that is for $e_{i}=\min _{j}\left\{e_{j}\right\}$, we have $\frac{e_{i}}{\sum_{j} e_{j}}<\frac{1}{n}$, in which case $\operatorname{Pr}\left\{\varepsilon_{i}=1\right\}<\frac{\bar{n}}{n}$.

Observation 3. The expectation of random variable $\varepsilon_{i}$ (which is 1 if the $i$ th person gets the better quality good and 0 otherwise), is given by ${ }^{10}$

$$
\mathrm{E}\left(\varepsilon_{i}\right)=1 \times \operatorname{Pr}\left(\varepsilon_{i}=1\right)+0 \times \operatorname{Pr}\left(\varepsilon_{i}=0\right)=P_{i} .
$$

Now $\sum_{i} \mathrm{E}\left(\varepsilon_{i}\right)=\mathrm{E}\left(\sum_{i} \varepsilon_{i}\right)=\mathrm{E}(\bar{n})=\bar{n}$. But $\sum_{i} \mathrm{E}\left(\varepsilon_{i}\right)=\sum_{i} P_{i}$. In this case too, we can verify that $\sum_{i=1}^{n} \operatorname{Pr}\left\{\varepsilon_{i}=1\right\}=\bar{n}$.

Observation 4. The probability of being successful is increasing in one's efforts (and decreasing on other's efforts). Hence

$$
\frac{\partial \operatorname{Pr}\left\{\varepsilon_{i}=1\right\}}{\partial e_{i}}=\frac{\bar{n}(n-\bar{n})(1-\lambda)}{(n-1)(\bar{n}+\lambda(n-\bar{n}))}\left[\frac{\sum_{j} e_{j}-e_{i}}{\left(\sum_{j} e_{j}\right)^{2}}\right]>0 .
$$

Hence if $e_{i}>e_{j}$, then $\operatorname{Pr}\left\{\varepsilon_{i}=1\right\}>\operatorname{Pr}\left\{\varepsilon_{j}=1\right\}$, i.e., if $i$ spends more effort than $j, i$ has more chance to find a seat than $j$.

\footnotetext{
${ }^{10} \mathrm{It}$ is easy to derive the variance of $\varepsilon_{i}$. It is as follows: Variance $\left\{\varepsilon_{i}\right\}=\mathrm{E}\left(\varepsilon_{i}-\mathrm{E}\left\{\varepsilon_{i}\right\}\right)^{2}=$ $\mathrm{E}\left(\varepsilon_{i}-P_{i}\right)^{2}=\mathrm{E}\left(\varepsilon_{i}^{2}+P_{i}^{2}-2 P_{i} \varepsilon_{i}\right)=P_{i}+P_{i}^{2}-2 P_{i}^{2}=P_{i}-P_{i}^{2}=P_{i}\left(1-P_{i}\right)=\operatorname{Pr}\left\{\varepsilon_{i}=\right.$ $0\} \operatorname{Pr}\left\{\varepsilon_{i}=1\right\}$.
} 
However, the rate of increase in probability is falling. That is,

$$
\frac{\partial^{2} \operatorname{Pr}\left\{\varepsilon_{i}=1\right\}}{\partial e_{i}^{2}}=-\frac{\bar{n}(n-\bar{n})(1-\lambda)}{(n-1)(\bar{n}+\lambda(n-\bar{n}))}\left[\frac{2\left(\sum_{j} e_{j}-e_{i}\right)}{\left(\sum_{j} e_{j}\right)^{3}}\right]<0 .
$$

Hence there is 'decreasing returns to effort' in the sense that increasing efforts increases the chances of procuring the better quality good but at a diminishing rate.

Observation 5. Suppose we are interested in the finding out how the probability of being successful changes as the weight given to efforts of people who are unsuccessful, $\lambda$, changes ${ }^{11}$. That is, we calculate $\frac{\partial \operatorname{Pr}\left\{\varepsilon_{i}=1\right\}}{\partial \lambda}$. Calculation yields the expression for this is given as follows:

$$
\frac{\partial \operatorname{Pr}\left\{\varepsilon_{i}=1\right\}}{\partial \lambda}=\frac{\bar{n}(n-\bar{n})}{(n-1)(\bar{n}+\lambda(n-\bar{n}))^{2}}\left(1-\frac{e_{i}}{\sum_{i=1}^{n} e_{i}} n\right)
$$

Hence the probability increases or decreases with $\lambda$, depending on individual effort $e_{i}$, relative to the the average $\frac{\sum_{i=1}^{n} e_{i}}{n}$. In particular, if $1>\frac{e_{i}}{\sum_{i=1}^{n} e_{i}} n$, that is $e_{i}<\frac{\sum_{i=1}^{n} e_{i}}{n}$, then $\frac{\partial \operatorname{Pr}\left\{\varepsilon_{i}=1\right\}}{\partial \lambda}>0$. (Similarly, if $1<\frac{e_{i}}{\sum_{i=1}^{n} e_{i}} n$, the opposite holds.)

This means that if individual $i$ is exerting less than average effort, then as desired, his probability of success falls, as $\lambda$ falls. Similarly, for someone exerting more than the average effort, the above derivative is negative, which means that his probability of success increases as $\lambda$ falls.

The last observation means that in our probability model, the smaller $\lambda$ gets, the more the probability of success of someone who is exerting above average effort, and lesser is the probability of success of someone who is exerting less than average effort. In the extreme case we have $\lambda=0$ which means the probability of an outcome is sensitive only to the efforts of those who have succeeded. So $\lambda=0$ should be a good simplification to work with, without sacrificing the essence of the model.

\footnotetext{
${ }^{11}$ Notice that in our probability model, the space $\Omega$ is essentially given by a collection $P_{\lambda}$ of non-negative real numbers $p_{\mathbf{v}, \lambda}$ allotted to each outcomes $\mathbf{v} \in \Omega$, satisfying the two conditions$$
p_{\mathbf{v}, \lambda} \geq 0, \quad \text { and } \quad \sum_{\mathbf{v} \in \Omega} p_{\mathbf{v}, \lambda}=1 .
$$

Hence for every $\lambda$, the pair $\left(\Omega, P_{\lambda}\right)$ is said to form a probability space. Now here we want to see if we can choose a particular $\lambda$ (and hence the corresponding probability space) to work with.
} 
Hereafter therefore, we work with the model assuming $\lambda=0$ (all results hold for the general case with positive $\lambda$, but only $\lambda=0$ is presented here to avoid cumbrous presentations). The following corollary lays down the probability in this case.

Corollary 1. If $\lambda=0$, we get

$$
\operatorname{Pr}\left\{\varepsilon_{i}=1\right\}=\frac{\bar{n}-1}{n-1}+\frac{e_{i}}{\sum_{i} e_{i}} \frac{n-\bar{n}}{n-1} .
$$

Find an example of the above probability model in Appendix A. We can also deduce the following from above:

Observation 6. In case of a single prize, the probability of success of the $i$ th individual reduces to the classic Tullock CSF (obtained by substituting $\bar{n}=1$ in $(6)$ ), given by

$$
\operatorname{Pr}\left\{\varepsilon_{i}=1\right\}=\frac{e_{i}}{\sum_{i} e_{i}}
$$

II(iv). Modelling cost of competing through effort exertion. Let there be $\bar{n}$ available prizes (which we synonymously use as the better quality good, like seats in a bus) and $n$ people competing for it, for example, consider a public transportation vehicle (for example, a bus) with $n$ people on board and which has a seating capacity of $\bar{n}$ seats, with $\bar{n}<n$. Let $\bar{c}$ be the fixed cost associated with consuming the better quality good/prize (like the cost of travelling while being seated which could be the fatigue experienced), and $g(n-\bar{n})$ be the cost of availing the low quality option, that is cost incurred if no prize is won (like the cost of travelling while standing) which could potentially depend on the total number of people who are availing this option. Both $\bar{c}$ and $g($.$) are same$ for all individuals and hence have no $i$ subscript. Assume $g($.$) has the property$ that $\partial g(.) / \partial n \geq 0$ which means that the cost of availing the low quality option cannot go down with greater number of people availing it. Also $\bar{c}<g(0)$, that is the cost of availing the better quality option is less than the cost of availing the low quality option, even when nobody is availing it (like it is better to sit, even when there is nobody standing inside a public transport vehicle), so that going for the better quality product is always preferable.

Now let the probability of a person getting the better quality good or not, be derived from the previous probability model. Hence let $P_{i}$ denote the probability that a person gets the better quality good (that is $\operatorname{Pr}\left\{\varepsilon_{i}=1\right\}$ ), like 
a person getting to sit inside a public transport vehicle, while $1-P_{i}$ is the remaining probability of a person having to settle for the lesser quality option (that is $\operatorname{Pr}\left\{\varepsilon_{i}=0\right\}$ ), like a person having to travel while standing inside a public transport vehicle.

In the random probability model, $P_{i}=\bar{n} / n$, while in the model with sequential availability $P_{i}=1$ for the first $\bar{n}$ people boarding and $P_{i}=0$ for the rest $(n-\bar{n})$ people. However the probability that a person finally gets the better quality good (like getting to sit in a congested bus), depends on the effort he or she puts in to get the good. Hence we make the probabilities a function of the efforts of all the people in the race, according to the preceding model, so that $P_{i}$, in our model will be given by (6).

Since exertion of effort is costly, let the cost of effort be given by $\chi_{i} \frac{e_{i}^{\alpha+1}}{\alpha+1}, \chi_{i}>$ $0, \alpha>-1$, so that the marginal cost of effort is positive and increasing, (which means the cost of effort increases at an increasing rate with increase in effort). Hence total expected cost of travelling (participating on the contest) in this model becomes

$$
C_{i}\left(e_{i}, e_{-i} ; n\right)=P_{i} \bar{c}+\left(1-P_{i}\right) g(n-\bar{n})+\chi_{i} \frac{e_{i}^{\alpha+1}}{\alpha+1}
$$

where $e_{-i}=\left(e_{1}, \ldots e_{i-1}, e_{i+1}, \ldots e_{n}\right)$. Next we turn to analysis of our model.

\section{EQUILIBRIUM ANALYSIS}

Every individual $i$ minimizes the expected cost of obtaining the good. Hence the FOC for minimization is given by (see the following details):

$$
\frac{\partial C_{i}(n)}{\partial e_{i}}=\frac{\partial P_{i}}{\partial e_{i}}(\bar{c}-g(n-\bar{n}))+\chi_{i} e_{i}^{\alpha}=0,
$$

where $\frac{\partial P_{i}}{\partial e_{i}}$ is given by

$$
\frac{\partial \operatorname{Pr}\left\{\varepsilon_{i}=1\right\}}{\partial e_{i}}=\frac{(n-\bar{n})}{(n-1)}\left[\frac{\sum_{j} e_{j}-e_{i}}{\left(\sum_{j} e_{j}\right)^{2}}\right]>0 .
$$

Consider the symmetric case, that is $\chi_{i}=\chi, \forall i$, in which every individual chooses the same equilibrium level of effort, call it $e^{*}$ (see Appendix $\mathrm{C}$ for the 
asymmetric case $\left.{ }^{12}\right)$. Then $e^{*}$ must satisfy

$$
\frac{(n-\bar{n})}{(n-1)}\left[\frac{1}{n e^{*}}-\frac{e^{*}}{n^{2} e^{* 2}}\right](\bar{c}-g(n-\bar{n}))+\chi e^{* \alpha}=0,
$$

which yields

$$
e^{*}=\left[\frac{1}{\chi}\left(\frac{n-\bar{n}}{n^{2}}\right)(g(n-\bar{n})-\bar{c})\right]^{\frac{1}{\alpha+1}} .
$$

The following proposition establishes that (9) is a unique Nash equilibrium of the congestion game in effort as given by (8).

Proposition 2. Consider the congestion game in effort as given in (8). If cost of effort is convex, (that is, $\alpha>0$ ), then there exists a unique Nash equilibrium in efforts. Moreover if $\chi_{i}=\chi, \forall i$, then the equilibrium effort is symmetric and it is given by:

$$
e^{*}=\left[\frac{1}{\chi}\left(\frac{n-\bar{n}}{n^{2}}\right)[g(n-\bar{n})-\bar{c}]\right]^{\frac{1}{\alpha+1}} .
$$

Proof: See Appendix B.

Note that equilibrium effort depends on cost differences $g(n-\bar{n})-\bar{c}$, and the number of people availing the lower quality option (standing, for example), $n-\bar{n}$. Greater is the relative discomfort of the lower quality option, relative to the better quality one, $g(n-\bar{n})-\bar{c}$, greater is the effort, while greater is the congestion, $n-\bar{n}$, greater is the effort. We do further comparative statics exercises as follows.

III(i). Analysis of equilibrium effort: Consider the symmetric Nash equilibrium outcome. We first check what happens to equilibrium effort with increasing congestion, that is with $n$ increasing. Assume $\alpha>-1$. Hence $e^{*}$

\footnotetext{
${ }^{12}$ Notice that in this model we have assumed that the impact of effort is the same for all individuals. However, the individuals may differ in their abilities to exert effort (one young passenger exerting one unit of effort may be more successful than a older one). Similarly one hour spent on studying by a clever student can be more effective than another. In other words, the same amount of work can be done by the cleverer student by expending lesser effort (that is the opportunity cost of effort is lower for the better quality student). Hence this formulation is very much reminiscent of cost differentials between types in (quality) signaling games (see the classic job market signaling paper Spence [27].) This idea is basically captured by the heterogeneity in the cost functions.
} 
increases (decreases) with $n$, if the following derivative is positive (negative):

$$
\begin{aligned}
& \frac{\partial}{\partial n}\left(\frac{n-\bar{n}}{n^{2}}(g(n-\bar{n})-\bar{c})\right) \\
= & \left(\frac{2 \bar{n}-n}{n^{3}}\right)(g(n-\bar{n})-\bar{c})+\left(\frac{n-\bar{n}}{n^{2}}\right) \frac{\partial g(.)}{\partial n} .
\end{aligned}
$$

Recall that $\frac{\partial g(.)}{\partial n}>0, n>\bar{n}$, and $g(n-\bar{n})>\bar{c}$. The following proposition summarizes the different cases.

Proposition 3. Consider the symmetric Nash equilibrium effort $e^{*}$ as given in Equation (9). Then $\frac{\partial e^{*}}{\partial n}>0$ if

$$
\left(\frac{2 \bar{n}-n}{n^{3}}\right)(g(n-\bar{n})-\bar{c})+\left(\frac{n-\bar{n}}{n^{2}}\right) \frac{\partial g(.)}{\partial n}>0
$$

whereas $\frac{\partial e^{*}}{\partial n}<0$, if the opposite inequality holds.

Moreover, if the cost of the low quality option, $g(n-\bar{n})$, is constant, and equal to $\bar{g}$, then: $\frac{\partial e^{*}}{\partial n}>0$ if $n<2 \bar{n} ; \frac{\partial e^{*}}{\partial n}=0$ if $n=2 \bar{n} ; \frac{\partial e^{*}}{\partial n}<0$ if $n>2 \bar{n}$.

Hence effort unambiguously increases as congestion increases if twice the number of seats is greater than the number of people on board.

Assuming $g(n-\bar{n})$ is a constant function of $n$, we get $\frac{\partial g(.)}{\partial n}=0$. Hence in this case

$$
\frac{\partial e^{*}}{\partial n}=\left(\frac{2 \bar{n}-n}{n^{3}}\right)(g(n-\bar{n})-\bar{c}) .
$$

The following graph lays out this case.

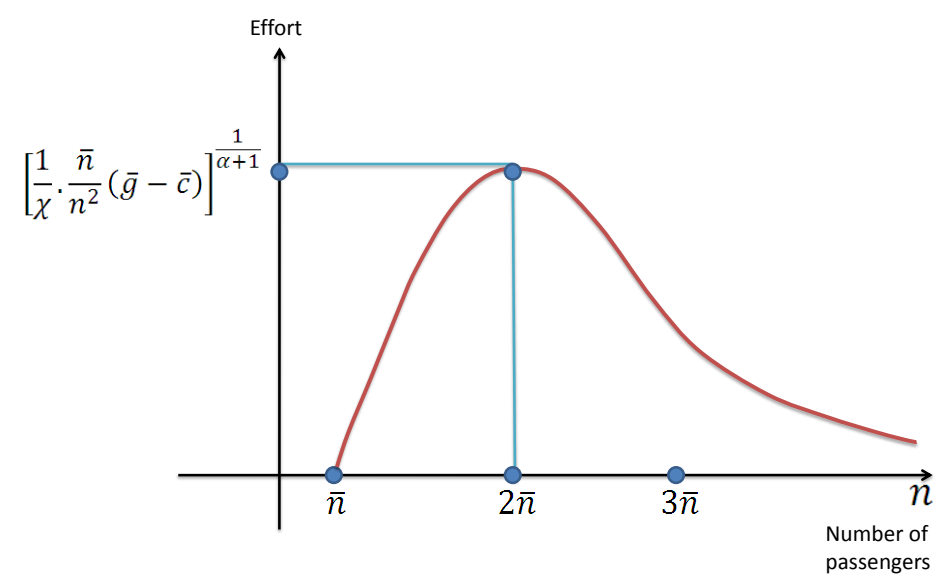

Figure 1: Change in effort with congestion 
In this case, for effort to be non-negative, we have $n>\bar{n}$, hence $e^{*}=0$ at $n=\bar{n}$. Effort rises till $n=2 \bar{n}$, and with even greater crowd, that is $n>2 \bar{n}$, effort falls with increasing $n$. Also

$$
\frac{\partial^{2} e^{*}}{\partial n^{2}}=(g(.)-\bar{c})\left(\frac{2}{n}-\frac{6 \bar{n}}{n^{2}}\right)
$$

so that $\frac{\partial^{2} e^{*}}{\partial n^{2}}<0$ for $n<3 \bar{n}$, and $\frac{\partial^{2} e^{*}}{\partial n^{2}}>0$ for $n>3 \bar{n}$. That is, the curve of effort is concave for $n<3 \bar{n}$ and convex thereafter.

Similarly we can derive other plausible comparative static effects as summarized in the following lemma.

Lemma 1. Consider the symmetric Nash equilibrium effort $e^{*}$ as given in Equation (9). The change in equilibrium effort with change in parameters $\bar{n}, \bar{c}$ and $\chi$ are as follows: $\frac{\partial e^{*}}{\partial \bar{n}}<0, \frac{\partial e^{*}}{\partial \bar{c}}<0, \frac{\partial e^{*}}{\partial \chi}<0$.

Hence we see that greater the number of available seats, lower will be equilibrium effort; the greater the discomfort of availing the better quality option (like the cost of travelling while seated), lower will be equilibrium effort; the higher the cost of exerting effort, lower will be equilibrium effort.

III(ii). Analysis of total cost. Again consider the symmetric equilibrium effort as given in proposition 2. Substituting for effort (from (9)) and probability (which is $\frac{\bar{n}}{n}$ ), the total cost can be calculated as follows:

$$
C_{i}^{*}(n)=\bar{c} \frac{\bar{n}}{n}+g(n-\bar{n})\left(1-\frac{\bar{n}}{n}\right)+\frac{\chi}{(1+\alpha)}\left[\frac{1}{n^{2} \chi}(n-\bar{n})(g(n-\bar{n})-\bar{c})\right] .
$$

Letting $f(n)=\left(\frac{\bar{n}}{n}-\frac{n-\bar{n}}{(\alpha+1) n^{2}}\right)$, we see

$$
\begin{aligned}
C_{i}^{*}(n) & =f(n) \bar{c}+(1-f(n)) g(n-\bar{n}) \\
& =(1-f(n))(g(n-\bar{n})-\bar{c})+\bar{c} .
\end{aligned}
$$

We can calculate ${ }^{13} C_{i}^{*}>0$.

Notice that the expost cost of a seated person (of one availing the better quality option) is

$$
\bar{c}+\frac{n-\bar{n}}{(\alpha+1) n^{2}}(g(n-\bar{n})-\bar{c}) .
$$

\footnotetext{
${ }^{13}$ Notice that $1-f(n)=1-\frac{\bar{n}}{n}+\frac{n-\bar{n}}{(\alpha+1) n^{2}}>0$ since $\bar{n}<n$ so that $\frac{\bar{n}}{n}<1$. Also $g(n-\bar{n})-\bar{c}>0$, that is, cost of using the low quality option is bigger than that of the high quality option. Hence $C_{i}^{*}>0$.
} 
Similarly, that of a person standing (of someone availing the lower quality option) will be

$$
g(n-\bar{n})+\frac{n-\bar{n}}{(\alpha+1) n^{2}}(g(n-\bar{n})-\bar{c}) .
$$

Now, consider total expected costs as given in (10). Let $g($.$) , the cost of the$ low quality option, be a constant function of $n$. Then we have from (10),

$$
\frac{\partial C_{i}^{*}}{\partial n}=-f^{\prime}(n)(g(.)-\bar{c})
$$

Hence $\operatorname{sign} \frac{\partial C_{i}^{*}}{\partial n}$ is opposite of sign $f^{\prime}(n)$. Now

$$
f^{\prime}(n)=-\frac{\bar{n}}{n^{2}}-\frac{2 \bar{n}-n}{n^{3}(\alpha+1)} .
$$

We can compute that $f^{\prime}(n)<0$ if $n<2 \bar{n}$, and hence $\frac{\partial C_{i}^{*}}{\partial n}>0$. With $n>2 \bar{n}$, $f^{\prime}(n)<0$ if $\alpha>\frac{n-2 \bar{n}}{n \bar{n}}-1$ in which case $\frac{\partial C_{i}^{*}}{\partial n}>0$. Now $\alpha>\frac{n-2 \bar{n}}{n \bar{n}}-1$ reduces to

$$
\alpha+1>\frac{1}{\bar{n}}-\frac{2}{\bar{n}} \text {. }
$$

Recall that the condition for the convexity of the objective function in the symmetric case was $\alpha>-\frac{2}{n}$. Moreover, $1>\frac{1}{\bar{n}}($ for $\bar{n}>1)$. Hence the above condition is always true. Hence the equilibrium expected cost per person always rises with increased congestion.

We can also compute change in equilibrium expected cost with change in $\bar{n}$. Here

$$
\frac{\partial C_{i}^{*}}{\partial \bar{n}}=-\frac{\partial f(n)}{\partial \bar{n}}(g(.)-\bar{c})
$$

Like before, $(g()-.\bar{c})>0$. Hence $\operatorname{sign} \frac{\partial C_{i}^{*}}{\partial n}$ is opposite of $\operatorname{sign} \frac{\partial f(n)}{\partial \bar{n}}$. Also, here

$$
\frac{\partial f(n)}{\partial \bar{n}}=\frac{1}{n}+\frac{1}{n^{2}(\alpha+1)}>0 .
$$

Hence

$$
\frac{\partial C_{i}^{*}}{\partial \bar{n}}<0
$$

The following proposition summarizes the findings ${ }^{14}$ :

Proposition 4. Consider the symmetric Nash equilibrium effort $e^{*}$ as given in Equation (9). The associated equilibrium total cost increases with increase

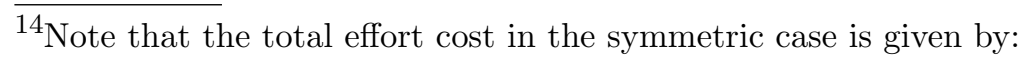

$$
n \chi \frac{e^{*(\alpha+1)}}{\alpha+1}=\left(\frac{n-\bar{n}}{n}\right)\left(\frac{g(.)-\bar{c}}{\alpha+1}\right) .
$$


in congestion, $n$, and decreases with increase in the better quality good. That $i s$,

$$
\begin{aligned}
& \frac{\partial C_{i}^{*}}{\partial n}>0 . \\
& \frac{\partial C_{i}^{*}}{\partial \bar{n}}<0 .
\end{aligned}
$$

III(iii). Analysis of total dissipation. 'Total dissipation', defined as $n e^{*}$, is the total effort exerted and dissipated due to congestion. As $n$ increases, there are more people exerting effort so there is an upward pressure on total dissipation, while each person is likely to exert lesser effort (as discussed above), which puts a downward pressure on total dissipation, hence a priori, it is not obvious in which direction total dissipation moves. After some rearrangements, the expression for total dissipation is given by

$$
n e^{*}=n\left[\frac{n-\bar{n}}{n^{2}}\right]^{\frac{1}{\alpha+1}}\left[\frac{g(n-\bar{n})-\bar{c}}{\chi}\right]^{\frac{1}{\alpha+1}} .
$$

Again for simplicity of calculations, let us assume, $g(n-\bar{n})$ is a constant function of $n$, and hence $\left[\frac{g(n-\bar{n})-\bar{c}}{\chi}\right]^{\frac{1}{\alpha+1}}$ becomes a constant, call it $L$. So the expression for change in total dissipation with change in $n$ is given by

$$
\frac{\partial\left(n e^{*}\right)}{\partial n}=L\left[\left(\frac{n-\bar{n}}{n^{2}}\right)^{\frac{1}{\alpha+1}}+n \frac{1}{\alpha+1}\left(\frac{n-\bar{n}}{n^{2}}\right)^{\frac{1}{\alpha+1}-1}\left(\frac{2 \bar{n}-n}{n^{3}}\right)\right] \text {. }
$$

Here $\frac{\partial\left(n e^{*}\right)}{\partial n}>0$ when $n \alpha>\bar{n}(\alpha-1)$, while $\frac{\partial\left(n e^{*}\right)}{\partial n}<0$ when $n \alpha<\bar{n}(\alpha-1)$. The following proposition summarizes the findings.

Proposition 5. Consider the symmetric Nash equilibrium effort $e^{*}$ as given in Equation (9). We have the following cases: $\frac{\partial\left(n e^{*}\right)}{\partial n}>0$, if $n \alpha>\bar{n}(\alpha-1) ; \frac{\partial\left(n e^{*}\right)}{\partial n}<0$, if $n \alpha<\bar{n}(\alpha-1)$.

Notice that when $\alpha>0$, we get $\frac{\partial\left(n e^{*}\right)}{\partial n}>0$, if $n>\bar{n}\left(1-\frac{1}{\alpha}\right) ; \frac{\partial\left(n e^{*}\right)}{\partial n}<$ 0 , if $n<\bar{n}\left(1-\frac{1}{\alpha}\right)$. However, the reverse condition holds for $\alpha<0$. In this case we $\operatorname{get}^{15} \frac{\partial\left(n e^{*}\right)}{\partial n}>0$, if $n<\bar{n}\left(1-\frac{1}{\alpha}\right) ; \frac{\partial\left(n e^{*}\right)}{\partial n}<0$, if $n>\bar{n}\left(1-\frac{1}{\alpha}\right)$.

\footnotetext{
${ }^{15}$ Also the cut-off $\bar{n}\left(1-\frac{1}{\alpha}\right)$ tends to $2 \bar{n}$ as $\alpha$ tends to -1 . However since $\alpha>-\frac{2}{n}>-1$ for $n>2$, the cut-off is actually bounded away from $2 \bar{n}$ (which is the relevant cut-off for changes in equilibrium effort).
} 
Diagrammatically, we obtain the change in total cost and change in total dissipation with congestion as follows:

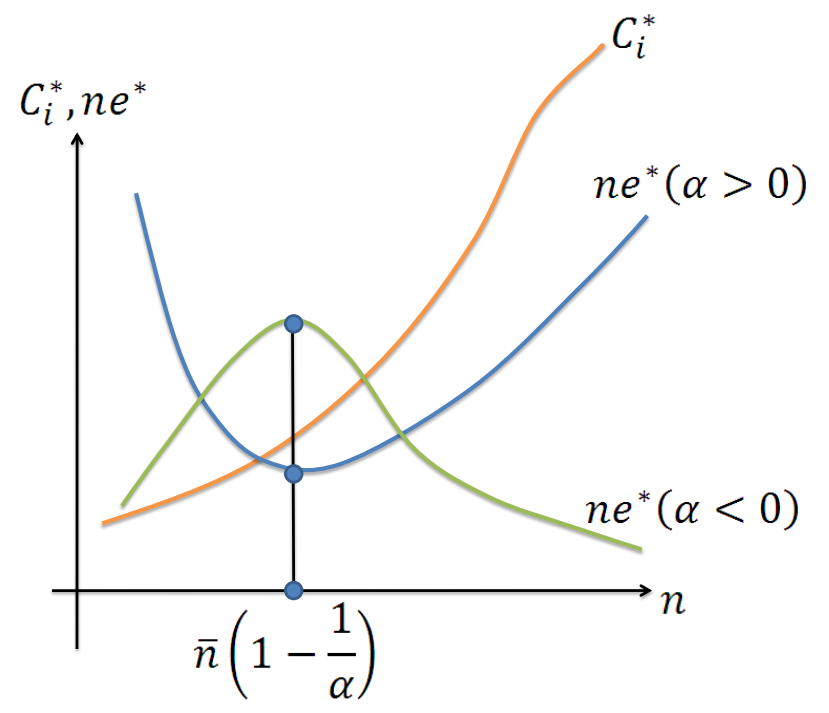

Figure 2: Change in individual cost and total dissipation with $n$

III(iv). Analysis of number of 'seats/prizes'. Suppose the social planner contemplates on increasing the number of seats in a public transport vehicle, or seats in a coveted educational institute. This will correspond to increasing $\bar{n}$, the better quality good, in our model. Let the per unit cost of $\bar{n}$ be given as $\pi$ so that total cost of providing $\bar{n}$ units of the better quality good is $\pi \bar{n}$.

Moreover, let the 'space' (or resources) occupied by one unit of the better quality good be $J$ (this could be literally the space occupied by a seat within a bus, or classroom space, campus accommodation, etc. for a 'seat' in a coveted educational institute). Hence the total amount of 'space' occupied by the better quality good is $J \bar{n}$. Let $S$ be the total capacity (depending on the context, this could be the total available space in a vehicle or total number of seats in all educational institutes). Let also $S$ be fixed (as is likely to be true in the short run). Hence the amount of 'space' for the lower quality good is $S-J \bar{n}$. Moreover feasibility implies $S \geq J \bar{n}$.

It is also plausible that the quality associated with the low-quality good falls as more and more of the better quality good is provided. That is, essentially, with limited total capacity, it is possible that providing for more of one quality 
good reduces the quality of the other. For example, as more seats are provided, the space available to stand in the bus falls, and even with the same amount of congestion, the 'disutility' or cost of travelling by standing, can increase. Similarly, if more seats are provided for elite educational institutes, it is possible for resources (teaching, administrative, housing facilities, etc.) to be allocated more towards those institutes so that the quality of education in the lesser quality institutes suffer.

In terms of the model, let $g=g(n-\bar{n}, S-J \bar{n})$ with $g($.$) varying inversely$ with $S-J \bar{n}$. Let us assume a simple functional form of $g($.$) as follows { }^{16}$ :

$$
g(n-\bar{n}, S-J \bar{n})=\frac{n-\bar{n}}{S-J \bar{n}} .
$$

with $S>J \bar{n}$. Hence the problem for the planner would be to find $\bar{n}$ that minimizes total costs which is the sum of costs of all individuals travelling as well as costs of increasing $\bar{n}$. That is, the planner minimizes:

$$
\sum_{i=1}^{n} C_{i}+\pi \bar{n}
$$

Substituting all the values in the cost function, we get

$$
\sum_{i=1}^{n} C_{i}=\bar{n} \bar{c}+\frac{(n-\bar{n})^{2}}{S-J \bar{n}}+\frac{(n-\bar{n})}{n(\alpha+1)}\left[\frac{n-\bar{n}}{S-J \bar{n}}-\bar{c}\right] .
$$

Hence the objective function for the planner becomes

$$
\sum_{i=1}^{n} C_{i}+\pi \bar{n}=\bar{n} \bar{c}+\frac{(n-\bar{n})^{2}}{S-J \bar{n}}+\frac{(n-\bar{n})}{n(\alpha+1)}\left[\frac{n-\bar{n}}{S-J \bar{n}}-\bar{c}\right]+\pi \bar{n} .
$$

The following proposition lays down the optimal number of seats.

Proposition 6. Consider the symmetric Nash equilibrium effort $e^{*}$ as given in Equation (9). Let $S, n$ be large and let $J(\pi+\bar{c})<1$ hold. Also let $\underline{n}=\frac{S}{J}(\sqrt{1-J(\pi+\bar{c})}+1)$. Then the first-order approximate solution of

\footnotetext{
${ }^{16}$ Note that, for this form, $\frac{d g(.)}{d \bar{n}}=\frac{J n-S}{(S-J \bar{n})^{2}}$. Having more seats has two consequences. If $J \bar{n}<S<J n$ (the problem of congestion arises when $S<J n$, that is the total space required for all the users is not available), then $d g(.) / d \bar{n}>0$, since providing more seats decreases the number of standing users, and thus increases congestion of standing users. On the other hand, if $J \bar{n}<J n<S$, then total space available is more than that required by all the users and hence the problem of congestion does not arise. In this case $d g(.) / d \bar{n}<0$, since more seats decrease the number of standing users, and thus reduce congestion of standing users.
} 
the optimal number of seats, $\bar{n}^{*}$ is given by:

$$
\bar{n}^{*}= \begin{cases}n & \text { if } n \leq \frac{S}{J} \\ \frac{S}{J}-\left(n-\frac{S}{J}\right) \frac{1}{\sqrt{1-J(\pi+\bar{c})}}>0, & \text { if } \frac{S}{J} \leq n \leq \underline{n} \\ 0, & \text { if } n \geq \underline{n} .\end{cases}
$$

Proof: See Appendix D.

Hence if the number of agents competing is too great, then it is optimal not to have any better quality good (like seats in a train etc.). But if it is not, then we should have a positive optimal amount of seats. The following graph shows the change in optimal number of seats with change in congestion.

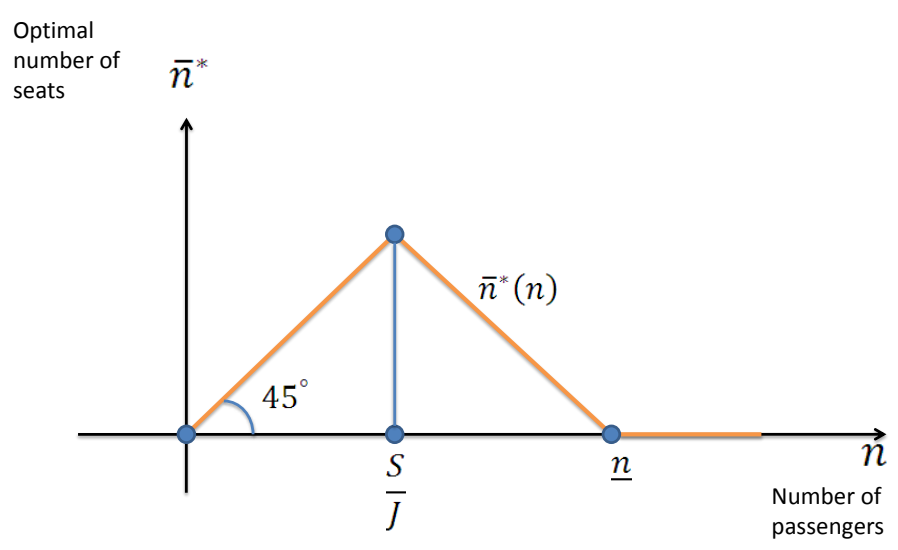

Figure 3: Change in optimal number of seats with change in $n$

Since $\frac{S}{J} \leq n \leq \underline{\mathrm{n}}$ is the interesting range, we analyse this further in the following proposition which lays down some interesting comparative statics.

Proposition 7. Consider the optimal number of seats $\bar{n}^{*}$ as given in Proposition 6. Then the following hold:

(a) $\bar{n}^{*}$ decreases with $\pi$; (b) $\bar{n}^{*}$ decreases with $n$; (c) $\bar{n}^{*}$ decreases with $\bar{c}$; (d) $\bar{n}^{*}$ decreases with $J$; (e) $\bar{n}^{*}$ decreases with $S$.

Proof: See Appendix E.

This supports the intuition that the optimal number of seats, $\bar{n}^{*}$ falls as $\pi, \bar{c}$, $J$, or $n$ increases (ceteris paribus). As $\pi$ increases, it means the per unit cost of providing the better quality good increases (like cost of wood for providing 
additional seats in a bus, cost of administrative and other support for extra seat in an educational institute etc.), and hence everything else same, it is optimal to provided less of that good.

Similarly, as $\bar{c}$ increases, it means the cost associated with consuming the better quality good is increasing (like the fatigue experienced in travelling by sitting or the opportunity cost faced by students while studying at elite educational institutes), in which case the optimal units of the better quality good should fall.

Again, $J$ increasing implies the 'space' occupied by one unit of the better quality good increasing (like space occupied by a seat in a bus, or the housing, classroom and other spaces occupied by a student in an elite educational institute), in which case, the optimal number of units of the better quality good should go down.

Also the provision of better quality goods going down when $n$ increases, or congestion increases. The possible explanation could be that with increasing $n$ the goal of the planner is to provide as many units of the good as possible to the consumers but since the better quality good involves more space and cost, it is optimal to reduce the supply of such better quality goods and increase that of the inferior quality goods. This explains why there are not too many seats in a bus in crowded countries like India - the goal is to accommodate as many people as possible, even if they are standing, in order to provide transportation to maximum number of people.

However, as total capacity $S$ increases (for example, if the size of the interior of a bus increases, or new educational institutes come up), the optimal number of seats increases.

\section{Model With Different EFFORTS FOR DIFFERENT tYPES OF GOODS}

Let there be $n$ people wanting to buy a unit of the good - either low or high quality (assume there are two kinds of prizes - a good one and a not-so-good one). Let $\bar{n}$ be the number of units available of the higher quality good. Hence only $\bar{n}$ number of people get the better quality good, while the remaining $n-\bar{n}$ people either consume the low quality good or do not consume any good at 
all. Let $\varepsilon_{i}$ be the possible outcome for every individual. Then

$$
\varepsilon_{i}= \begin{cases}-1, & \text { if } i \text { does not get any good at all } \\ 0, & \text { if } i \text { gets the low quality good } \\ 1, & \text { if } i \text { gets the higher quality good. }\end{cases}
$$

Hence the space of all outcomes $\Omega$ will be given by

$$
\Omega=\left\{\bar{v}=\left(\varepsilon_{1}, \ldots, \varepsilon_{n}\right): \varepsilon_{i}=-1,0,1 ; \sum_{i} \max \left\{\varepsilon_{i}, 0\right\}=\bar{n}\right\} .
$$

Let $e_{i l}$ be the effort exerted by the $i$ th person to get the low quality good, and $e_{i h}$ be the effort exerted by the $i$ th person to get the higher quality good.

Assumption 2. "Holistic" Approach with Dual Efforts: Let probability $p_{\bar{v}}$ of outcome $\bar{v}$ be proportional to the sum of the efforts of the people who are successful in obtaining the better quality good in the outcome $\bar{v}$ as well as the efforts of the people who are successful in obtaining the inferior quality good in the outcome $\bar{v}$. That is

$$
p_{\bar{v}} \propto\left\{\sum_{\substack{\bar{v} \in \Omega \\ \varepsilon_{i}(\bar{v})=0}} e_{i l}+\sum_{\substack{\bar{v} \in \Omega \\ \varepsilon_{i}(\bar{v})=1}} e_{i h}\right\} .
$$

Again, notice that this formulation is similar to the basic model with $\lambda=0$, since the people who do not finally get any good, do not contribute to the probability of any outcome. This is just a simplifying assumption since this modest addition leads to a sufficiently complicated model.

The formulation means that if $i$ does not get any good, $\varepsilon_{i}=-1$, and his contribution to both the terms is 0 . If $i$ gets the lower quality good, then $\varepsilon_{i}=0$, so that his contribution to the first expression is $e_{i l}$ while that to the second expression is 0 , so that his total contribution is his effort of getting the low quality good $e_{i l}$. If $i$ gets the higher quality good, then $\varepsilon_{i}=1$, and his contribution is $e_{i h}$.

If $\mathcal{K}$ is the constant of proportionality, then

$$
p_{\bar{v}}=\mathcal{K} \times\left\{\sum_{\substack{\bar{v} \in \Omega \\ \varepsilon_{i}(\bar{v})=0}} e_{i l}+\sum_{\substack{\bar{v} \in \Omega \\ \varepsilon_{i}(\bar{v})=1}} e_{i h}\right\} .
$$


The following lemma lays down the value of $\mathcal{K}$.

Lemma 2. Let $\sum_{i} e_{i l}=e_{L}$ and $\sum_{i} e_{i h}=e_{H}$. Then $\mathcal{K}$ is given by

$$
\mathcal{K}=\frac{1}{\left(\begin{array}{l}
n \\
\bar{n}
\end{array}\right) 2^{(n-\bar{n}-1)}\left\{e_{L}\left(\frac{n-\bar{n}}{n}\right)+2 \frac{\bar{n}}{n} e_{H}\right\}} .
$$

where $e_{i l}$ is the effort exerted by the ith person to get the low quality good, and $e_{i h}$ be the effort exerted by the ith person to get the higher quality good.

Proof: See Appendix F.

Let us now compute the probability that a particular person, say 1, has obtained the low quality good, that is $\varepsilon_{1}=0$.

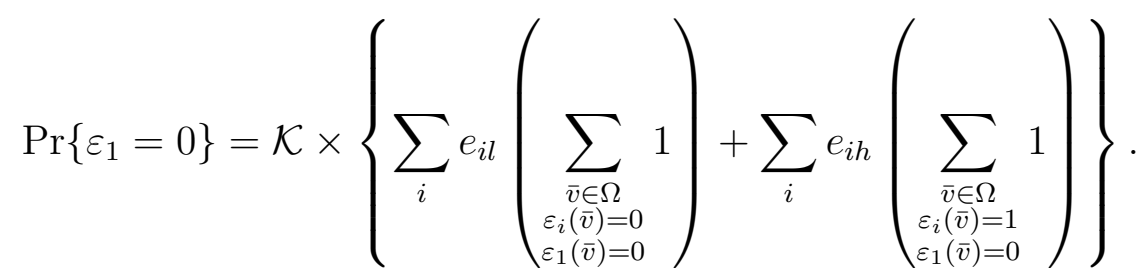

Now calculating as before, we get the following:

$$
\begin{aligned}
& \sum_{\substack{\bar{v} \in \Omega \\
\varepsilon_{i}(\bar{v})=0 \\
\varepsilon_{1}(\bar{v})=0}} 1= \begin{cases}\left(\begin{array}{c}
n-1 \\
\bar{n}
\end{array}\right) \times 2^{(n-1-\bar{n})} & \text { if } i=1 \\
\left(\begin{array}{c}
n-2 \\
\bar{n}
\end{array}\right) \times 2^{(n-2-\bar{n})} & \text { if } i \neq 1,\end{cases} \\
& \sum_{\substack{\bar{v} \in \Omega \\
\varepsilon_{i}(\bar{v})=1 \\
\varepsilon_{1}(\bar{v})=0}} 1= \begin{cases}0 \text { if } i=1 \\
\left(\begin{array}{c}
n-2 \\
\bar{n}-1
\end{array}\right) \times 2^{(n-1-\bar{n})} & \text { if } i \neq 1 .\end{cases}
\end{aligned}
$$

Substitution and some lengthy calculations yield:

$$
\operatorname{Pr}\left\{\varepsilon_{1}=0\right\}=\frac{n-\bar{n}}{2(n-1)}+\frac{n-\bar{n}}{2(n-1)} \times \frac{e_{1 l}(n+\bar{n}-1)-e_{L}-2 \bar{n} e_{1 h}}{e_{L}(n-\bar{n})+2 \bar{n} e_{H}} .
$$

Hence for any individual $i$, the following proposition lays down the expression of the probability for a person to obtain the low quality good:

Proposition 8. The probability that person $i$ has obtained the low quality good is

$$
\operatorname{Pr}\left\{\varepsilon_{i}=0\right\}=\frac{n-\bar{n}}{2(n-1)}+\frac{n-\bar{n}}{2(n-1)} \times \frac{e_{i l}(n+\bar{n}-1)-e_{L}-2 \bar{n} e_{i h}}{e_{L}(n-\bar{n})+2 \bar{n} e_{H}}
$$


We can also calculate the probability that person 1 has obtained the higher quality good, that is $\varepsilon_{1}=1$, as follows:

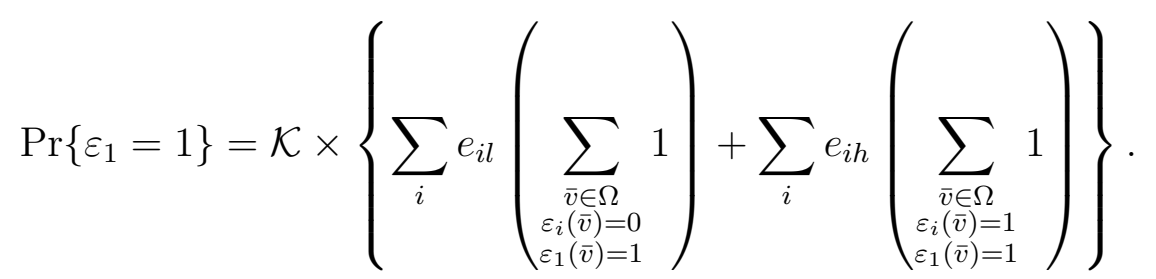

Calculating, we get the following:

$$
\begin{aligned}
& \sum_{\substack{\bar{v} \in \Omega \\
\varepsilon_{i}(\bar{v})=0 \\
\varepsilon_{1}(\bar{v})=1}} 1=\left\{\begin{array}{l}
0 \text { if } i=1 \\
\left(\begin{array}{l}
n-2 \\
\bar{n}-1
\end{array}\right) \times 2^{(n-1-\bar{n})} \text { if } i \neq 1,
\end{array}\right.
\end{aligned}
$$

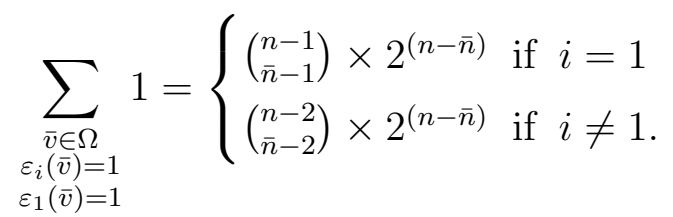

Again, substitution and some lengthy calculations yield:

$$
\operatorname{Pr}\left\{\varepsilon_{1}=1\right\}=\frac{(n-\bar{n}) e_{L}+2(n-\bar{n}) e_{1 h}-(n-\bar{n}) e_{1 l}+2(\bar{n}-1) e_{H}}{(n-1)\left(e_{L} \frac{n-\bar{n}}{\bar{n}}+2 e_{H}\right)} .
$$

Hence for any individual $i$, the following proposition summarizes the probability of a person to obtain the better quality option:

Proposition 9. The probability that person $i$ obtains the higher quality option is

$$
\operatorname{Pr}\left\{\varepsilon_{i}=1\right\}=\frac{(n-\bar{n}) e_{L}+2(n-\bar{n}) e_{i h}-(n-\bar{n}) e_{i l}+2(\bar{n}-1) e_{H}}{(n-1)\left(e_{L} \frac{n-\bar{n}}{\bar{n}}+2 e_{H}\right)}
$$

Let $P_{i h}$ be the probability of getting the higher quality good (that is $\operatorname{Pr}\left\{\varepsilon_{i}=\right.$ $1\}), P_{i l}$ be the probability of getting the lower quality good (that is $\operatorname{Pr}\left\{\varepsilon_{i}=0\right\}$ ), and $\left(1-P_{i l}-P_{i h}\right)$ is the remaining probability of not getting any good at all.

Hence, in the symmetric case, with $e_{i l}=e_{l}, \forall i$ and $e_{i h}=e_{h}, \forall i$, we get, the probability of a person getting the better quality good, $P_{h}$ and that of a person getting the poorer quality good $P_{l}$ (notice there is no $i$ subscript since it is the 
same for all $i$ ), as follows:

$$
\begin{aligned}
P_{l} & =\frac{n-\bar{n}}{2(n-1)}+\frac{(n-\bar{n})}{2 n(n-1)} \frac{e_{l}(\bar{n}-1)-2 \bar{n} e_{h}}{e_{l}(n-\bar{n})+2 \bar{n} e_{h}} \\
P_{h} & =\frac{\bar{n}}{n} .
\end{aligned}
$$

Observation 7 . Notice that $P_{h}$ is just as in the random probability model. That is, the probability that one gets the higher quality good when everybody exerts equal effort to get the higher and the lesser quality goods, is given as in the random probability model by $\frac{\bar{n}}{n}$.

Modelling cost of travelling: Let each individual $i$ exert effort $e_{i h}$ for the high-quality good and $e_{i l}$ be the effort the individual exerts for the low-quality good (like exam preparation etc. for getting into elite educational institutes, and that for getting into other institutes, in the context of education, or effort to only board and stand or board and sit, in the context of travelling in public transportation).

If individual $i$ gets the better quality good (after having exerted effort $e_{i h}$ for the high quality good and $e_{i l}$ for the low quality good good), his cost is (assume for simplicity that $\chi_{i h}=\chi_{h}, \forall i$ and $\left.\chi_{i l}=\chi_{l}, \forall i\right)$

$$
\bar{c}+\chi_{h} \frac{e_{i h}^{\alpha_{h}+1}}{\alpha_{h}+1}+\chi_{l} \frac{e_{i l}^{\alpha_{l}+1}}{\alpha_{l}+1} .
$$

Similarly, if individual $i$ gets the lower quality good after having exerted effort $e_{i h}$ and $e_{i l}$, his cost is (for simplicity, we assume $g(n-\bar{n})=\bar{g}$, a constant function, and not varying with the level of congestion)

$$
\bar{g}+\chi_{h} \frac{e_{i h}^{\alpha_{h}+1}}{\alpha_{h}+1}+\chi_{l} \frac{e_{i l}^{\alpha_{l}+1}}{\alpha_{l}+1} .
$$

Let $\tau$ be the cost of not being able to get any good at all (like the time cost of delay if one misses a bus, or the psychological cost of not being admitted to any educational institute for higher studies and so on). So if individual $i$ fails to get any good, after having exerted effort to obtain either type of the good, his cost is

$$
\tau+\chi_{h} \frac{e_{i h}^{\alpha_{h}+1}}{\alpha_{h}+1}+\chi_{l} \frac{e_{i l}^{\alpha_{l}+1}}{\alpha_{l}+1} .
$$

Moreover since we assume that getting a good is always preferable to not getting any good, it must be true that $\bar{c} \leq \bar{g} \leq \tau$. Hence the expected cost for 
an individual $i$ is

$$
C_{i}=P_{i h} \bar{c}+P_{i l} \bar{g}+\left(1-P_{i h}-P_{i l}\right) \tau+\chi_{h} \frac{e_{i h}^{\alpha_{h}+1}}{\alpha_{h}+1}+\chi_{l} \frac{e_{i l}^{\alpha_{l}+1}}{\alpha_{l}+1} .
$$

The following proposition lays down the cost-minimizing effort levels:

Proposition 10. Consider the congestion game with two types of efforts, namely effort $e_{h}$ for the high-quality good and effort $e_{l}$ for the low quality good. Moreover, consider linear cost of effort, that is, $\alpha_{l}=\alpha_{h}=0, \forall i$. Then the cost-minimizing effort levels are given by

$$
\begin{gathered}
e_{l}^{*}=0 \\
e_{h}^{*}=\frac{n-\bar{n}}{2 n^{2} \chi_{h}}(\tau-2 \bar{c}+\bar{g}) .
\end{gathered}
$$

Proof. The F.O.Cs for interior optimum efforts will be as follows:

$$
\begin{aligned}
\frac{\partial C_{i}}{\partial e_{i h}} & =\frac{\partial P_{i h}}{\partial e_{i h}}(\bar{c}-\tau)+\frac{\partial P_{i l}}{\partial e_{i h}}(\bar{g}-\tau)+\chi_{h} e_{i h}^{\alpha_{h}} \\
\frac{\partial C_{i}}{\partial e_{i l}} & =\frac{\partial P_{i h}}{\partial e_{i l}}(\bar{c}-\tau)+\frac{\partial P_{i l}}{\partial e_{i l}}(\bar{g}-\tau)+\chi_{l} e_{i l}^{\alpha_{l}} .
\end{aligned}
$$

The change in probabilities are as follows (assuming $e_{i h}=e_{h}, \forall i, e_{i l}=e_{l}, \forall i$ ):

$$
\begin{aligned}
& \left.\frac{\partial P_{i h}}{\partial e_{i h}}\right|_{e_{i h}=e_{h}}=\frac{2(n-\bar{n})}{e_{i l}=e_{l}}= \\
& \left.\frac{\partial P_{i h}}{\partial e_{i l}}\right|_{e_{i h}=e_{h}}=-\frac{n-\bar{n}}{e_{i l}=e_{l}}=-\frac{n-\bar{n}}{n^{2}\left(e_{l} \frac{n-\bar{n}}{\bar{n}}+2 e_{h}\right)} \\
& \left.\frac{\partial P_{i l}}{\partial e_{i h}}\right|_{e_{i h}=e_{h}}=-\frac{\bar{n}(n-\bar{n})\left(e_{l}(n-\bar{n}+1)+2 \bar{n} e_{h}\right)}{n^{2}\left(e_{l}(n-\bar{n})+2 \bar{n} e_{h}\right)^{2}} \\
& \left.\frac{\partial P_{i l}}{\partial e_{i l}}\right|_{e_{i h}=e_{h}}=\frac{(n-\bar{n})\left(e_{l}(n-\bar{n})(n+\bar{n}-1)+2 \bar{n} e_{h}(n+\bar{n})\right)}{2 n^{2}\left(e_{l}(n-\bar{n})+2 \bar{n} e_{h}\right)^{2}} .
\end{aligned}
$$

Substituting the above in the F.O.Cs and simplifying we get the following expressions:

$$
\begin{aligned}
& -2 \bar{n}(n-\bar{n})(\tau-\bar{c})\left(e_{l}(n-\bar{n})+2 \bar{n} e_{h}\right) \\
& -(\bar{g}-\tau) \bar{n}(n-\bar{n})\left(e_{l}(n-\bar{n}+1)+2 \bar{n} e_{h}\right)+\chi_{h} e_{h}^{\alpha_{h}} n^{2}\left(e_{l}(n-\bar{n})+2 \bar{n} e_{h}\right)^{2}=0 \\
& 2 \bar{n}(n-\bar{n})(\tau-\bar{c})\left(e_{l}(n-\bar{n})+2 \bar{n} e_{h}\right) \\
& +(\bar{g}-\tau) \bar{n}(n-\bar{n})\left(e_{l}(n-\bar{n})(n+\bar{n}-1)+2 \bar{n} e_{h}(n+\bar{n})\right) \\
& +2 \chi_{l} e_{l}^{\alpha_{l}} n^{2}\left(e_{l}(n-\bar{n})+2 \bar{n} e_{h}\right)^{2}=0 .
\end{aligned}
$$


Letting $\alpha_{l}=\alpha_{h}=0$, the F.O.Cs reduce to the following:

$$
\begin{gathered}
\quad-2 \bar{n}(n-\bar{n})(\tau-\bar{c})\left(e_{l}(n-\bar{n})+2 \bar{n} e_{h}\right) \\
-(\bar{g}-\tau) \bar{n}(n-\bar{n})\left(e_{l}(n-\bar{n}+1)+2 \bar{n} e_{h}\right) \\
+\chi_{h} n^{2}\left(e_{l}(n-\bar{n})+2 \bar{n} e_{h}\right)^{2}=0 . \\
2 \bar{n}(n-\bar{n})(\tau-\bar{c})\left(e_{l}(n-\bar{n})+2 \bar{n} e_{h}\right) \\
+(\bar{g}-\tau) \bar{n}(n-\bar{n})\left(e_{l}(n-\bar{n})(n+\bar{n}-1)+2 \bar{n} e_{h}(n+\bar{n})\right)
\end{gathered}
$$$$
+2 \chi_{l} n^{2}\left(e_{l}(n-\bar{n})+2 \bar{n} e_{h}\right)^{2}=0 .
$$

Notice that (21) gives the cost-minimizing choice of $e_{h}$ for any given $e_{l}$. Similarly (22) gives the cost-minimizing choice of $e_{l}$ for any given $e_{h}$. We can check from the FOCs that for any $e_{h} \geq 0$, the solution of $e_{l}$ turns out to be negative. Hence the only feasible $e_{l}=0$. Hence for $e_{l}=0$, the equilibrium effort for the high quality good can be solved to be

$$
e_{h}^{*}=\frac{n-\bar{n}}{2 n^{2} \chi_{h}}(\tau-2 \bar{c}+\bar{g}) .
$$

We can check that the SOCs are also satisfied at these values.

Notice that (20) is very similar to the optimal effort in the basic model (instead of $(g()-.\bar{c})$, now we have the average of $(g()-.\bar{c})$ and $(\tau-\bar{c})$, with $g()=.\bar{g}$, and also $\alpha=0)$. Hence all the comparative statics of the basic model will carry through.

Interestingly we see that even when we allow for multiple types of goods, this model yields that positive effort will only be exerted to obtain the better quality good ${ }^{17}$. Hence the finding rationalizes the demand for seats in public transport as well as great competition for gaining seats in elite educational institutes.

\section{Extension: Two Different Populations}

Let us assume there are two distinct subgroups in the population, group $a$ and group $b$ (we could think of them as an 'old' group and a 'young' group, or 'men' and 'women', or 'local' and 'tourists' etc.). Let a generic group be

\footnotetext{
${ }^{17}$ This result is reminiscent of the usual result in signaling games, the Spence education model, for example, where in a separating equilibrium, the 'bad/low' type of a worker exerts the minimum possible or 0 level of effort.
} 
denoted by $r, r=a, b$. Let the expected cost of travelling of an individual in group $r$ be given as follows:

$$
C_{r}(n)=P_{r} \bar{c}_{r}+\left(1-P_{r}\right) g_{r}(n-\bar{n})+\chi_{r} \frac{e_{r}^{\alpha_{r}+1}}{\alpha_{r}+1}, \quad r=a, b,
$$

where $P_{r}$ is the probability that a member of group $r$ gets to sit in the bus, and it is the same for all members in this group. Here $P_{r}$ will be given as follows:

$$
P_{r}=\frac{\bar{n}-1}{n-1}+\left[\frac{e_{r}}{n_{a} e_{a}+n_{b} e_{b}}\right] \frac{n-\bar{n}}{n-1}, r=a, b
$$

where $n_{r}$ is the number of people in group $r$, such that $n_{a}+n_{b}=n$.

Now the FOC for minimization for group $a$ is given by (assuming an interior optimum exists):

$$
\frac{\partial C_{a}(n)}{\partial e_{a}}=\frac{\partial P_{a}}{\partial e_{a}}\left(\bar{c}_{a}-g(n-\bar{n})\right)+\chi_{a} e_{a}^{\alpha_{a}}=0
$$

where

$$
\frac{\partial P_{a}}{\partial e_{a}}=\left[\frac{n-\bar{n}}{n-1}\right]\left[\frac{n_{b} e_{b}}{\left(n_{a} e_{a}+n_{b} e_{b}\right)^{2}}\right] .
$$

Hence the FOCs for cost minimization of the two groups will be as follows (assuming interior optimum):

$$
\begin{aligned}
& \frac{n-\bar{n}}{n-1}\left[\frac{n_{b} e_{b}}{\left(n_{a} e_{a}+n_{b} e_{b}\right)^{2}}\right]\left(g_{a}(n-\bar{n})-\bar{c}_{a}\right)=\chi_{a} e_{a}^{\alpha_{a}} \\
& \frac{n-\bar{n}}{n-1}\left[\frac{n_{a} e_{a}}{\left(n_{a} e_{a}+n_{b} e_{b}\right)^{2}}\right]\left(g_{b}(n-\bar{n})-\bar{c}_{b}\right)=\chi_{b} e_{b}^{\alpha_{b}}
\end{aligned}
$$

which are two equations in two unknowns $e_{a}$ and $e_{b}$ and can be solved for the equilibrium values of the efforts of the two groups. Dividing the first FOC by the second and letting $\alpha_{a}=\alpha_{b}=\alpha$, we get, relative effort as follows:

$$
\frac{e_{a}}{e_{b}}=\left[\frac{\frac{g_{a}(n-\bar{n})-\bar{c}_{a}}{n_{a} \chi_{a}}}{\frac{g_{b}(n-\bar{n})-\bar{c}_{b}}{n_{b} \chi_{b}}}\right]^{\frac{1}{\alpha+1}} \text {. }
$$

This yields the expected insight that the relative effort of group $a,\left(e_{a} / e_{b}\right)$ increases as $\left(\chi_{b} / \chi_{a}\right)$ rises, that is the cost of effort falls in one's own group relative to the other. Also, for fixed $n,\left(e_{a} / e_{b}\right)$ falls as $\left(n_{b} / n_{a}\right)$ increases, that is as its own group size falls relative to the other group's size. Moreover $\left(e_{a} / e_{b}\right)$ rises as $\left[\left(g_{a}(n-\bar{n})-\bar{c}_{a}\right) /\left(g_{b}(n-\bar{n})-\bar{c}_{b}\right)\right]$ increases, that is the cost of consuming 
the lower quality good increases more, relative to that of consuming the better quality good in one's own group, compared to the other.

\section{Conclusion}

In this paper, we have introduced a new ("holistic") approach to evaluate success probabilities in multi-player, multi-prize contests. In this formulation, we start from the probability of the aggregate outcome, and then derive individual probabilities of success from it. It turns out that in the case of a single prize, the generalization we obtain, boils down to the individual probability of success of the classic CSF introduced by Tullock (see Observation 6).

We then use this probability to study optimal effort exertion in the presence of scarcity, that is, when the number of players is strictly greater than the number of prizes. Moreover, we have introduced certain other complexities in our setting. For example, in our setting, the high quality good (prize) is subject to scarcity, while the low quality good (which a player will consume by default if he fails to get the prize) is subject to congestion. However, it is always preferable to get the higher quality good, even if the other lower quality good is not subject to congestion. That is, a player may or may not obtain a unit of the prize, while no player can be excluded from the low quality good. In addition, there is congestion faced by users of the low quality good as the number of such users increase.

Our analysis yields that when costs of effort are symmetric among players, then a symmetric Nash equilibrium exists in which all players exert the same level of effort, and this effort first increases and then falls with congestion (see Fig. 1). The associated total cost increases with congestion while the total dissipation could first fall and then increase or first increase and then fall with congestion, depending on parametric restrictions (see Fig. 2). In this case, we also analyse the optimal number of prizes as a matter of policy instrument for the public authorities (see Fig. 3).

As an analysis of scarce resource facing excessive demand, the proposed approach is amenable to different contexts like getting admissions into elite educational institutions, procuring seats in congested public transportation, or getting allotments in regulated housing markets, among others. In all these 
situations, prices do not clear the market and agents exert effort to have access to the limited number of high quality good (prizes).

We have also envisaged an extension of the basic model in this paper. Here we study effort exertion with two different types of goods, a better quality and an inferior quality good. In this case too, we begin with a "holistic" approach, but with dual efforts, and derive success probabilities of individuals gaining access to any one of these two goods. If an agent does not get the high or the low quality good, he has access to a third alternative (worse than getting any of the two goods). We show that in equilibrium, all effort will be devoted to having access to the better quality alternative.

Instead of two, in principle, multiple quality prizes that can be obtained with competitive effort, could be modelled with our framework, but the computations get that much more complex, without necessarily adding to the insights. Since the model computes the probability that an individual has access to a good (of a particular quality), it is straightforward to introduce risk aversion in the approach (where the risk represents the risk of not finding a proper place). Preliminary results show that, as expected, equilibrium effort (in the symmetric case) increases with increasing risk aversion.

Empirical investigation of the model is definitely an interesting avenue of future research. To this end, and keeping in mind the transportation example, we have just completed the administration of an internet survey (called Mimetic) in Paris, where we assess transport mode choice, departure time choice, value of time and aversion to risk. For those choosing the public transport, we also ask questions about their willingness to pay, for a seat, or for less congestion; commuters typically have the option of finding a seat, standing with low density congestion and standing with a high density crowd. This will help us to get approximate values of the parameters of our model and hence help us to test the accuracy of predictions for levels of equilibrium effort, cost, dissipation and other variables of interest. Being able to assess the amount of effort (and being able to monetarize it), provides a new dimension to the cost benefit analysis. In such a manner, it will be possible to assess the congestion cost in public transportation, a topic which has been neglected so far. Similarly, we can apply the method for measuring the cost of effort in other situations (such as education, or the regulated housing market). 
The framework we have developed has potentially other applications, outside those mentioned so far. Consider for example the marriage market, with the number of potential grooms (players) higher than that of potential brides (prizes). Not allowing for polygamy (so that a prize cannot be shared) and assuming the same 'quality' of the brides (same "attractiveness", for example), we are in a situation just like in our model, essentially a multi-player, multi-prize contest. Employing our approach therefore, we can find the equilibrium efforts exerted by the potential grooms (efforts at convincing and wooing the few women). Hence our approach provides an alternative solution to the Beckman-Koopmans standard matching model used in the economics of the family (see Chiappori, McCann and Nesheim [5]).

The cluster-based heuristic interpretation of our probability model (see Section II(ii)) is particularly applicable in this case. Here, it means that a few men would concentrate their effort to seduce the same woman. Note that the choice of the cluster can be strategic, although this has not been modelled so far.

However, when the quality of the prizes differ (like in the more realistic situation of different women being of varying appeal), the situation is more involved. The women who are perceived to be too "attractive" may generate a level of effort among the competitors that is socially too high (causing total dissipation and total cost to rise). On the other hand, with asymmetric costs of efforts, the individuals who exert more effort, are likely to be determined and more reliable (indicating lower cost of exerting effort and hence being better as a potential grooms), so that effort may also provide a useful signal.

However in the present paper, we have not explored the (more realistic) situation of both multiple-quality prizes, as well as asymmetric costs of effort exertion. These questions will be the topic for future research.

\section{References}

[1] Anderson, Simon P., de Palma, André, and Thisse, Jacques-François. 1992. Discrete Choice Theory of Product Differentiation. The MIT Press.

[2] Arnott, Richard, de Palma, André, Lindsey, Robin. 1993. A Structural Model of PeakPeriod Congestion: A Traffic Bottleneck with Elastic Demand, The American Economic Review, Vol. 83, No. 1, pp. 161-179. 
[3] de Palma, André, Picard, Nathalie, Waddell, Paul. 2007. Discrete choice models with capacity constraints: An empirical analysis of the housing market of the greater Paris region, Journal of Urban Economics, Vol. 62, No. 2, pp. 204-230.

[4] Blavatskyy, Pavlo R. 2010. Contest success function with the possibility of a draw: Axiomatization. Journal of Mathematical Economics, Vol. 46, 267 - 276.

[5] Chiappori, Pierre-André, McCann Robert, and Nesheim, Lars. 2010. Hedonic price equilibria, stable matching, and optimal transport: equivalence, topology, and uniqueness. Economic Theory, Vol. 42, 317 - 354.

[6] Clark, Derek J. and Riis, Christian. 1998. Competition over More Than One Prize, The American Economic Review, Vol. 88, No. 1, 276 - 289.

[7] Clark, Derek J. and Riis, Christian. 1998. Contest success functions: An extension. Economic Theory, Vol. 11, 201 - 204.

[8] Chowdhury, Subhasish M. and Sheremeta, Roman M. March 2010. A generalized Tullock contest, preprint.

[9] Dixit, Avinash. 1987. Strategic Behavior in Contests, The American Economic Review, Vol. 77, No. 5, 891 - 898.

[10] Gaudry M. J. I. and Dagenais M. G. The dogit model, Transportation Research, Vol. 13B, No. 2, 105 - 112.

[11] Gradstein, Mark and Nitzan, Shmuel. 1989. Advantageous multiple rent seeking, Mathematical Modelling, Vol. 12, 511 - 518.

[12] Hillman, A. L., and Riley, J. G. 1989. Politically Contestable Rents and Transfers, Economics and Politics, Vol. 1, 1739.

[13] Hirshleifer, Jack. 1989. Conflict and rent-seeking success functions: ratio vs. difference models of relative success. Public Choice, Vol. 63, 101 - 112.

[14] Hwang, Sung-Ha. Contest Success Functions: Theory and Evidence, preprint.

[15] Laffont, Jean-Jacques and Martimort, David. 2005. The Theory of Incentives: The Principal-Agent Model. Princeton University Press.

[16] Lam, William H.K., Cheung Chung-Yu, Lam, C.F. A study of crowding effects at the Hong Kong light rail transit stations, Transportation Research Part A, Vol. 33, 401 415.

[17] Martimort, David and Stole, Lars. 2011. Aggregate Representations of Aggregate Games with Applications to Common Agency, preprint.

[18] Mohring, Herbert. 1972. Optimization and Scale Economies in Urban Bus Transportation, The American Economic Review, Vol. 62, No. 4, pp. 591-604.

[19] Moldovanu, Benny and Sela, Aner. 2001. Optimal Allocation of Prizes in Contests. The American Economic Review, Vol. 91, No. 3, 542-558.

[20] Münster, Johannes. 2009. Group contest success functions, Economic Theory, Vol. 41, $345-357$.

[21] Nitzan, Shmuel. 1994. Modelling rent-seeking contests, European Journal of Political Economy, Vol. 10, 41 - 60.

[22] Nti, Kofi O. 1997. Comparative statics of contests and rent-seeking games, International Economic Review, 38, 43-59. 
[23] Parry, I. W. H and Small, K. H. 2009. Should urban transit subsidies be reduced? American Economic Review, Vol. 99 (3), 700 -724.

[24] Pucher, John, Korattyswaroopam, Nisha, and Ittyerah, Neenu. 2004. The Crisis of Public Transport in India: Overwhelming Needs but Limited Resources, Journal of Public Transportation, Vol. 7, No. 4.

[25] Rosen, Sherwin. 1986. Prizes and incentives in elimination tournaments, American Economic Review, 76, 701 - 709 .

[26] Skaperdas, Stergios. 1996. Contest success functions, Economic Theory, Vol. 7, 283 290.

[27] Spence, Michael. 1973. Job Market Signaling, The Quarterly Journal of Economics, Vol. 87, No. 3, pp. 355-374.

[28] Szymanski, Stefan. 2003. The Economic Design of Sporting Contests. Journal of Economic Literature, 41, 1137-1187.

[29] Tullock, Gordon. 1980. Efficient rent seeking. In James M. Buchanan, Robert D. Tollison, Gordon Tullock, (Eds.), Toward a theory of the rent-seeking society. College Station, TX: Texas A\&M University Press, 97-112.

[30] Vickrey, William. 1963. Pricing in Urban and Suburban Transport, American Economic Review, Papers and Proceedings, Vol. 53, 452-65.

[31] Vickrey, William. 1969. Congestion Theory and Transport Investment, American Economic Review, Papers and Proceedings, Vol. 59, 251-61. 


\section{Appendix}

(The appendix has been divided into two parts - Appendices A, B, C, D, E, $\mathrm{F}$ have been referred to in the main body of the paper, and hence have been placed under 'Appendix for Online Publication', while appendices G and H have not, and hence have been placed under 'Appendix Not Necessary for Online Publication'. Appendix G illustrates on the correlation of outcomes in the basic model and appendix $\mathrm{H}$ draws a parallel of our probability model with a simple lottery.)

\section{Appendix For Online Publication}

\section{Appendix A. Derivation of the probability model With an}

\section{EXAMPLE}

Consider the basic probability model with $\lambda=0$. Let $n=3, \bar{n}=2$. Then the possible outcomes and associated probabilities are as follows:

$$
\begin{aligned}
& \bar{v}_{1}=(1,1,0): p_{1}=K\left(\varepsilon_{1}\left(\bar{v}_{1}\right) e_{1}+\varepsilon_{2}\left(\bar{v}_{1}\right) e_{2}+\varepsilon_{3}\left(\bar{v}_{1}\right) e_{3}\right)=K\left(e_{1}+e_{2}\right) \\
& \bar{v}_{2}=(1,0,1): p_{2}=K\left(\varepsilon_{1}\left(\bar{v}_{2}\right) e_{1}+\varepsilon_{2}\left(\bar{v}_{2}\right) e_{2}+\varepsilon_{3}\left(\bar{v}_{2}\right) e_{3}\right)=K\left(e_{1}+e_{3}\right) \\
& \bar{v}_{3}=(0,1,1): p_{3}=K\left(\varepsilon_{1}\left(\bar{v}_{3}\right) e_{1}+\varepsilon_{2}\left(\bar{v}_{3}\right) e_{2}+\varepsilon_{3}\left(\bar{v}_{3}\right) e_{3}\right)=K\left(e_{2}+e_{3}\right)
\end{aligned}
$$

Hence summing over all outcomes we get,

$$
\begin{aligned}
1=p_{1}+p_{2}+p_{3} & =K * \sum_{i=1}^{3} e_{i}\left(\sum_{\bar{v} \in \Omega} \varepsilon_{i}(\bar{v})\right) \\
& =K *\left(e_{1}\left(\sum_{\bar{v} \in \Omega} \varepsilon_{1}(\bar{v})\right)+e_{2}\left(\sum_{\bar{v} \in \Omega} \varepsilon_{2}(\bar{v})\right)+e_{3}\left(\sum_{\bar{v} \in \Omega} \varepsilon_{3}(\bar{v})\right)\right)
\end{aligned}
$$

Now:

$$
\begin{aligned}
& \sum_{\bar{v} \in \Omega} \varepsilon_{1}(\bar{v})=\sum_{\substack{\bar{v} \in \Omega \\
\varepsilon_{1}(\bar{v})=1}} 1=2 \\
& \sum_{\bar{v} \in \Omega} \varepsilon_{2}(\bar{v})=\sum_{\substack{\bar{v} \in \Omega \\
\varepsilon_{2}(\bar{v})=1}} 1=2 \\
& \sum_{\bar{v} \in \Omega} \varepsilon_{3}(\bar{v})=\sum_{\substack{\bar{v} \in \Omega \\
\varepsilon_{3}(\bar{v})=1}} 1=2
\end{aligned}
$$


Hence substituting we get

$$
1=K * 2 *\left(e_{1}+e_{2}+e_{3}\right)
$$

And $2=\left(\begin{array}{l}3 \\ 2\end{array}\right)$, for this example.

Now consider an example with $n=3, \bar{n}=2, e_{1}=8, e_{2}=e_{3}=1$. Then we get the following:

$$
\begin{aligned}
& P_{1}=\frac{1}{2}+\frac{8}{10} \frac{1}{2}=\frac{18}{20} \\
& P_{2}=\frac{1}{2}+\frac{1}{10} \frac{1}{2}=\frac{11}{20} \\
& P_{3}=\frac{1}{2}+\frac{1}{10} \frac{1}{2}=\frac{11}{20}
\end{aligned}
$$

Again the sum equals $\bar{n}=2$. This means (roughly) that person 1 exerting high effort gets to sit with a very high probability in one of the seats, while the other two people exerting the same low probability gets to sit with almost equal probability in the remaining 1 seat.

\section{Appendix B. Proof of Proposition 2}

Proof. We can write the objective function as:

$$
C_{i}\left(e_{i}, e_{-i} ; n\right)=g(n-\bar{n})+[\bar{c}-g(n-\bar{n})] P_{i}+\chi_{i} \frac{e_{i}^{\alpha+1}}{\alpha+1} .
$$

Now $P_{i}=\frac{\bar{n}-1}{n-1}+\frac{n-\bar{n}}{n-1} \frac{e_{i}}{\sum_{j} e_{j}}$

Therefore, we can rewrite the objective function as

$$
\begin{aligned}
C_{i}\left(e_{i}, e_{-i} ; n\right) & =g(n-\bar{n})+[\bar{c}-g(n-\bar{n})]\left[\frac{\bar{n}-1}{n-1}\right] \\
& +[\bar{c}-g(n-\bar{n})]\left[\frac{n-\bar{n}}{n-1}\right]\left[\frac{e_{i}}{\sum_{j} e_{j}}\right]+\chi_{i} \frac{e_{i}^{\alpha+1}}{\alpha+1} .
\end{aligned}
$$

Consider a change of variable $e_{i}=\exp \left(E_{i}\right)$, so that

$$
\mathbb{P}_{i}=\frac{\exp \left(E_{i}\right)}{\sum_{j} \exp \left(E_{j}\right)} .
$$

Also let

$$
\begin{gathered}
\widehat{c}=\left[g(n-\bar{n}) \frac{n-\bar{n}}{n-1}+\bar{c} \frac{\bar{n}-1}{n-1}\right], \\
\omega=[g(n-\bar{n})-\bar{c}] \frac{n-\bar{n}}{n-1} .
\end{gathered}
$$


Hence the objective function can be written:

$$
C_{i}\left(E_{i}, E_{-i} ; n\right)=\widehat{c}-\omega \mathbb{P}_{i}+\frac{\chi_{i}}{\alpha+1} \exp \left[(\alpha+1) E_{i}\right]
$$

Therefore, the F.O.C.s are:

$$
\frac{\partial C_{i}\left(E_{i}, E_{-i} ; n\right)}{\partial E_{i}}=-\omega \mathbb{P}_{i}\left(1-\mathbb{P}_{i}\right)+\chi_{i} \exp \left[(\alpha+1) E_{i}\right]=0
$$

Thus equation (24) defines the best reply of agent $i$, with respect to the strategy of the other agents, $E_{i}^{b r}$.

Now assuming $\chi_{i}=\chi \forall i$, we get the symmetric Nash equilibrium as follows (refer to appendix $\mathrm{C}$ for the asymmetric solution):

$$
\exp \left[E^{*}\right]=e^{*}=\left[\frac{\omega}{\chi}\left(\frac{n-1}{n^{2}}\right)\right]^{\frac{1}{\alpha+1}}
$$

or

$$
e^{*}=\left[\frac{1}{\chi}\left(\frac{n-\bar{n}}{n^{2}}\right)[g(n-\bar{n})-\bar{c}]\right]^{\frac{1}{\alpha+1}} .
$$

The S.O.C.s are (which are true even for the asymmetric case):

$$
\frac{\partial^{2} C_{i}\left(E_{i}, E_{-i} ; n\right)}{\partial E_{i}^{2}}=-\omega\left(1-2 \mathbb{P}_{i}\right) \mathbb{P}_{i}\left(1-\mathbb{P}_{i}\right)+\chi_{i}(\alpha+1) \exp \left[(\alpha+1) E_{i}\right] .
$$

Substituting the F.O.C. in the S.O.C., we get,

$$
\begin{aligned}
\left.\frac{\partial^{2} C_{i}\left(E_{i}, E_{-i} ; n\right)}{\partial E_{i}^{2}}\right|_{\text {f.o.c. }} & =-\omega\left(1-2 \mathbb{P}_{i}\right) \mathbb{P}_{i}\left(1-\mathbb{P}_{i}\right)+(\alpha+1) \omega \mathbb{P}_{i}\left(1-\mathbb{P}_{i}\right) \\
& =\omega \mathbb{P}_{i}\left(1-\mathbb{P}_{i}\right)\left[-\left(1-2 \mathbb{P}_{i}\right)+(\alpha+1)\right] \\
& =\omega \mathbb{P}_{i}\left(1-\mathbb{P}_{i}\right)\left[2 \mathbb{P}_{i}+\alpha\right]
\end{aligned}
$$

For convexity of the objective function, we need the above expression to be positive. Therefore, a sufficient condition would be $\left[2 \mathbb{P}_{i}+\alpha\right]>0$, for any $\mathbb{P}_{i}$. For this, $\alpha>0$ is a sufficient condition.

In the symmetric case, the condition becomes, $\left[\frac{2}{n}+\alpha\right]>0$ which reduces to $\alpha>-\frac{2}{n}$. For very large $n$, this implies again that the cost function is convex.

Uniqueness. The condition for uniqueness is

$$
\sum_{\substack{j=1 \ldots N \\ j \neq i}}\left|\frac{\partial E_{i}^{b r}}{\partial E_{j}}\right|<1
$$


Let

$$
\Omega_{i}=-\omega \mathbb{P}_{i}\left(1-\mathbb{P}_{i}\right)+\chi_{i} \exp \left[(\alpha+1) E_{i}\right]=0 .
$$

Thus, by the implicit function theorem, we get,

$$
\begin{aligned}
\frac{\partial E_{i}^{b r}}{\partial E_{j}} & =-\frac{\partial \Omega_{i} / \partial E_{j}}{\partial \Omega_{i} / \partial E_{i}} \\
= & -\frac{\omega\left(2 \mathbb{P}_{i}-1\right) \mathbb{P}_{i} \mathbb{P}_{j}}{-\omega\left(1-2 \mathbb{P}_{i}\right) \mathbb{P}_{i}\left(1-\mathbb{P}_{i}\right)+\chi_{i}(\alpha+1) \exp \left[(\alpha+1) E_{i}\right]} \\
& =\frac{\mathbb{P}_{j}\left(2 \mathbb{P}_{i}-1\right)}{\left(1-\mathbb{P}_{i}\right)\left(2 \mathbb{P}_{i}+\alpha\right)} .
\end{aligned}
$$

Hence

$$
\left|\frac{\partial E_{i}^{b r}}{\partial E_{j}}\right|=\frac{\mathbb{P}_{j}\left|2 \mathbb{P}_{i}-1\right|}{\left(1-\mathbb{P}_{i}\right)\left(2 \mathbb{P}_{i}+\alpha\right)}
$$

Hence summing over all $j \neq i$, we get

$$
\sum_{\substack{j=1 \ldots N \\ j \neq i}}\left|\frac{\partial E_{i}^{b r}}{\partial E_{j}}\right|=\frac{\left|2 \mathbb{P}_{i}-1\right|}{2 \mathbb{P}_{i}+\alpha}
$$

which is a decreasing function of $\alpha$. Hence, for $\alpha=-1$,

$$
\sum_{\substack{j=1 \ldots N \\ j \neq i}}\left|\frac{\partial E_{i}^{b r}}{\partial E_{j}}\right|=1
$$

And $\forall \alpha>-1$,

$$
\sum_{\substack{j=1 \ldots N \\ j \neq i}}\left|\frac{\partial E_{i}^{b r}}{\partial E_{j}}\right|<1
$$

This proves uniqueness and the proposition.

\section{Appendix C. Asymmetric Case}

We can write the objective function as follows (notice $\bar{c}_{i}$ instead of $\bar{c}, g_{i}($. instead of $g(),. \alpha_{i}$ instead of $\alpha$ and $\chi_{i}$ instead of $\chi$, as costs for individual $i$ ):

Recall

$$
C_{i}\left(e_{i}, e_{-i} ; n\right)=g_{i}(n-\bar{n})+\left[\overline{c_{i}}-g_{i}(n-\bar{n})\right] P_{i}+\chi_{i} \frac{e_{i}^{\alpha_{i}+1}}{\alpha_{i}+1} .
$$

$$
P_{i}=\frac{\bar{n}-1}{n-1}+\frac{n-\bar{n}}{n-1} \frac{e_{i}}{\sum_{j} e_{j}}
$$


Like before, with $e_{i}=\exp \left(E_{i}\right)$ and

$$
\begin{gathered}
\mathbb{P}_{i}=\frac{\exp \left(E_{i}\right)}{\sum_{j} \exp \left(E_{j}\right)}, \\
\widehat{c_{i}}=\left[g_{i}(n-\bar{n}) \frac{n-\bar{n}}{n-1}+\overline{c_{i}} \frac{\bar{n}-1}{n-1}\right], \\
\omega_{i}=\left[g_{i}(n-\bar{n})-\overline{c_{i}}\right] \frac{n-\bar{n}}{n-1},
\end{gathered}
$$

we get

$$
C_{i}\left(E_{i}, E_{-i} ; n\right)=\widehat{c}_{i}-\omega_{i} \mathbb{P}_{i}+\frac{\chi_{i}}{\alpha_{i}+1} \exp \left[\left(\alpha_{i}+1\right) E_{i}\right] .
$$

Therefore, the F.O.C.s are:

$$
\frac{\partial C_{i}\left(E_{i}, E_{-i} ; n\right)}{\partial E_{i}}=-\omega_{i} \mathbb{P}_{i}\left(1-\mathbb{P}_{i}\right)+\chi_{i} \exp \left[\left(\alpha_{i}+1\right) E_{i}\right]=0 .
$$

That is,

$$
\omega_{i} \mathbb{P}_{i}\left(1-\mathbb{P}_{i}\right)=\chi_{i} \exp \left[\left(\alpha_{i}+1\right) E_{i}\right] .
$$

Let $\Phi=\sum_{j} \exp \left(E_{j}\right)=\sum_{j} e_{j}$. Then, we get

$$
\begin{aligned}
0 & =\omega_{i} \frac{\exp \left(E_{i}\right)}{\Phi}\left(1-\frac{\exp \left(E_{i}\right)}{\Phi}\right)-\chi_{i}\left[\exp \left(E_{i}\right)\right]^{\alpha_{i}+1} \\
& =\omega_{i} \exp \left(E_{i}\right)\left(\Phi-\exp \left(E_{i}\right)\right)-\Phi^{2} \chi_{i}\left[\exp \left(E_{i}\right)\right]^{\alpha_{i}+1}
\end{aligned}
$$

Since $e_{i}=\exp \left(E_{i}\right)$, the F.O.C. becomes:

$$
\begin{aligned}
\omega_{i}\left(\Phi-e_{i}\right) e_{i}-\Phi^{2} \chi_{i}\left(e_{i}\right)^{\alpha_{i}+1} & =0 \\
\Phi^{2} \chi_{i}\left(e_{i}\right)^{\alpha_{i}+1}+\omega_{i}\left(e_{i}\right)^{2}-\Phi \omega_{i} e_{i} & =0 .
\end{aligned}
$$

The first-order condition, simplifies as follows:

$$
\left(e_{i}\right)^{\alpha_{i}}+\frac{\omega_{i}}{\chi_{i}} \frac{e_{i}}{\Phi^{2}}-\frac{\omega_{i}}{\Phi \chi_{i}}=0
$$

If $\alpha_{i}=0 \forall i$ : We have $\frac{\omega_{i}}{\chi_{i}} \frac{e_{i}}{\Phi^{2}}=\frac{\omega_{i}}{\Phi \chi_{i}}-1$, or $\frac{\omega_{i}}{\chi_{i}}=\Phi-\Phi^{2} \frac{\chi_{i}}{\omega_{i}}$

$$
e_{i}=\left(1-\Phi \frac{\chi_{i}}{\omega_{i}}\right) \Phi
$$


Thus:

$$
\begin{aligned}
\sum_{j} e_{j} & =\Phi \sum_{j}\left(1-\Phi \frac{\chi_{i}}{\omega_{i}}\right)=\Phi\left(n-\Phi \sum_{j} \frac{\chi_{j}}{\omega_{j}}\right)=\Phi ; \\
\Phi & =\frac{(n-1)}{\sum_{j} \frac{\chi_{j}}{\omega_{j}}} \\
e_{i}^{*} & =\left(1-\frac{(n-1) \frac{\chi_{i}}{\omega_{i}}}{\sum_{j} \frac{\chi_{j}}{\omega_{j}}}\right) \frac{(n-1)}{\sum_{j} \frac{\chi_{j}}{\omega_{j}}} .
\end{aligned}
$$

The following proposition summarizes our findings in the asymmetric case.

Proposition 11. Consider asymmetric but linear cost of effort, that is, cost of effort of individual $i$ is given by $\chi_{i} e_{i}, \forall i$. Let $\omega_{i}=\left[g_{i}(n-\bar{n})-\overline{c_{i}}\right] \frac{n-\bar{n}}{n-1}$. Then the Nash equilibrium level of effort of individual $i$ is given by

$$
e_{i}^{*}=\left(1-\frac{(n-1) \frac{\chi_{i}}{\omega_{i}}}{\sum_{j} \frac{\chi_{j}}{\omega_{j}}}\right) \frac{(n-1)}{\sum_{j} \frac{\chi_{j}}{\omega_{j}}} .
$$

Note that $e_{r}^{*}>e_{s}^{*}$ iff $\frac{\chi_{s}}{\omega_{s}}>\frac{\chi_{r}}{\omega_{r}}$.

We can also compute equilibrium probabilities in this case to be as follows:

$$
P_{i}^{*}=1-\frac{(n-\bar{n}) \frac{\chi_{i}}{\omega_{i}}}{\sum_{j} \frac{\chi_{j}}{\omega_{j}}} .
$$

Notice that lower is the cost of exerting effort, $\frac{\chi_{i}}{\omega_{i}}$, relative to $\sum_{j} \frac{\chi_{j}}{\omega_{j}}$, the higher is the probability of finding a seat (please refer to footnote 11 in the paper for an interpretation of heterogeneity of cost functions in our context).

Moreover, note that if all cost of effort $\left(\chi_{i}\right)$ are multiplied by $\tau$, then each level of effort is divided by $\tau$, and the total cost of effort $\sum_{j} \chi_{j} e_{j}$ (for this case of $\left.\alpha_{i}=0\right)$ remains the same. Taking effort multiplicatively has no impact of consumers surplus, so that the tax revenue $(\tau-1) \sum_{j} e_{j}$ corresponds to the social benefit. 
We can verify that in the symmetric case, we have:

$$
\begin{aligned}
e^{*} & =\left(1-\frac{(n-1)}{n}\right) \frac{(n-1) \omega}{n \chi} \\
e^{*} & =\frac{\omega}{\chi}\left(\frac{n-1}{n^{2}}\right)=\frac{[g(n-\bar{n})-\bar{c}]}{\chi}\left(\frac{n-\bar{n}}{n^{2}}\right)
\end{aligned}
$$

as derived earlier.

\section{Appendix D. Proof of Proposition 6}

Proof. The planner minimizes the following objective function w.r.t $\bar{n}$ :

$$
\sum_{i=1}^{n} C_{i}+\pi \bar{n}=\bar{n} \bar{c}+\frac{(n-\bar{n})^{2}}{S-J \bar{n}}+\frac{(n-\bar{n})}{n(\alpha+1)}\left[\frac{n-\bar{n}}{S-J \bar{n}}-\bar{c}\right]+\pi \bar{n} .
$$

Differentiating w.r.t. $\bar{n}$, the F.O.C. is:

$$
\begin{aligned}
(\pi+\bar{c})(S-J \bar{n})^{2}- & 2(n-\bar{n})(S-J \bar{n})+(n-\bar{n})^{2} J \\
- & \frac{(S-J \bar{n})}{n(\alpha+1)}(n-\bar{n}-\bar{c}(S-J \bar{n}))+\frac{(n-\bar{n})(J n-S)}{n(\alpha+1)}=0 .
\end{aligned}
$$

This is a quadratic equation in $\bar{n}$ and can be written in the form $A \bar{n}^{2}+B \bar{n}+C=$ 0 where the coefficients are as follows:

$$
\begin{aligned}
& A=-J\left[-J(\pi+\bar{c})+1+\frac{1-J \bar{c}}{n(\alpha+1)}\right] \\
& B=2 S\left[-J(\pi+\bar{c})+1+\frac{1-J \bar{c}}{n(\alpha+1)}\right] \\
& C=(\pi+\bar{c}) S^{2}-2 n S+J n^{2}-\frac{2 S}{\alpha+1}+\frac{\bar{c} S^{2}}{n(\alpha+1)}+\frac{J n}{\alpha+1} .
\end{aligned}
$$

In general, it is difficult to get a closed-form solution to the above equation. Hence we let $n$ and $\mathrm{S}$ be large (as is plausible) and take first order approximations. This means we can approximate $-J(\pi+\bar{c})+1+\frac{1-J \bar{c}}{n(\alpha+1)}$ by $-J(\pi+\bar{c})+1$, as the last term with $n$ in the denominator is of smaller size, since $n \gg 1$. This results in the following first order approximations for $A$ and $B$ :

$$
\begin{aligned}
& \tilde{A}=-J[-J(\pi+\bar{c})+1] \\
& \tilde{B}=2 S[-J(\pi+\bar{c})+1] .
\end{aligned}
$$

To get the approximation for $C$, we see that out of the six terms the last three terms are of smaller size, e.g. the terms $\frac{2 S}{\alpha+1}, \frac{\bar{c} S^{2}}{n(\alpha+1)}$ are of smaller size compared to $(\pi+\bar{c}) S^{2}$, and $\frac{J n}{\alpha+1}$ is of smaller size compared to $J n^{2}$ (as $n \gg 1$ 
and $S \gg 1$ ). So it follows that the first order approximation for $C$ is given by

$$
\tilde{C}=(\pi+\bar{c}) S^{2}-2 n S+J n^{2} .
$$

Now substituting the first order approximations in the equation and solving, we get

$$
(S-J \bar{n})^{2}(J(\pi+\bar{c})-1)+(S-J n)^{2}=0 .
$$

So for any feasible solution, we must have $J(\pi+\bar{c})<1$. (This is plausible given $\pi, \bar{c}$, and $J$ are likely to be small and can be chosen appropriately). Hence we can solve $\bar{n}^{*}$ from above to be:

$$
\bar{n}^{*}=\frac{S}{J}-\left(n-\frac{S}{J}\right) \frac{1}{\sqrt{1-J(\pi+\bar{c})}} .
$$

(Notice that $J n>S$, otherwise $\bar{n}=n$ and the problem of congestion would not be relevant.) We can check that the S.O.C. for minimization also holds.

However, notice that if $n$ is very large, then $\bar{n}^{*}$ will become negative, so that the optimal $\bar{n}$ will be 0 . Hence we can solve for the cut-off of $n$ for feasible $\bar{n}$, by setting

$$
\frac{S}{J}-\left(n-\frac{S}{J}\right) \frac{1}{\sqrt{1-J(\pi+\bar{c})}}=0
$$

This solves for

$$
n=\frac{S}{J}(\sqrt{1-J(\pi+\bar{c})}+1)
$$

Call $n$ in (27), ‥ Hence

$$
\bar{n}^{*}= \begin{cases}0, & \text { if } n \geq \underline{\mathrm{n}} \\ \frac{S}{J}-\left(n-\frac{S}{J}\right) \frac{1}{\sqrt{1-J(\pi+\bar{c})}}>0, & \text { if } n<\underline{\mathrm{n}} .\end{cases}
$$




\section{Appendix E. Proof of Proposition 7}

Proof. Differentiating $\bar{n}^{*}$ we get the following:

$$
\begin{aligned}
\frac{\partial \bar{n}^{*}}{\partial \pi} & =-\left(n-\frac{S}{J}\right)\left(-\frac{1}{(\sqrt{1-J(\pi+\bar{c})})^{2}}\right)(-J)<0, \\
\frac{\partial \bar{n}^{*}}{\partial n} & =-\frac{1}{\sqrt{1-J(\pi+\bar{c})}}<0, \\
\frac{\partial \bar{n}^{*}}{\partial \bar{c}} & =-\left(n-\frac{S}{J}\right)\left(-\frac{1}{(\sqrt{1-J(\pi+\bar{c})})^{2}}\right)(-J)<0, \\
\frac{\partial \bar{n}^{*}}{\partial J} & =-\frac{S}{J^{2}}-\left[\frac{S}{J^{2}} \frac{1}{\sqrt{1-J(\pi+\bar{c})}}+\left(n-\frac{S}{J}\right)\left(-\frac{1}{(\sqrt{1-J(\pi+\bar{c})})^{2}}\right)(-(\pi+\bar{c}))\right], \\
& <0 \\
\frac{\partial \bar{n}^{*}}{\partial S} & =\frac{1}{J}+\frac{1}{J} \sqrt{1-J(\pi+\bar{c})}>0 .
\end{aligned}
$$

This proves the proposition.

\section{Appendix F. Proof of lemma 2: Derivation of $\mathcal{K}$}

Proof. Summing over all possible outcomes, we get

$$
\begin{aligned}
& 1=\sum_{\bar{v} \in \Omega} p_{\bar{v}}=\mathcal{K} \times\left\{\sum_{\bar{v} \in \Omega} \sum_{\substack{\bar{v} \in \Omega \\
\varepsilon_{i}(\bar{v})=0}} e_{i l}+\sum_{\bar{v} \in \Omega} \sum_{\substack{\bar{v} \in \Omega \\
\varepsilon_{i}(\bar{v})=1}} e_{i h}\right\} . \\
& 1=\mathcal{K} \times\left\{\sum_{i} e_{i l}\left(\sum_{\substack{\bar{v} \in \Omega \\
\varepsilon_{i}(\bar{v})=0}} 1\right)+\sum_{i} e_{i h}\left(\sum_{\substack{v \in \Omega \\
\varepsilon_{i}(\bar{v})=1}} 1\right)\right\} .
\end{aligned}
$$

(There is no effort counted for $\varepsilon_{i}=-1$.) Now $\left(\sum_{\substack{\bar{v} \in \Omega \\ \varepsilon_{i}(\bar{v})=0}} 1\right)$ counts the number of vectors of length $(n-1)$ (since the $i$ th person's outcome is known which is to get the low quality good), where there are $\bar{n}$ number of 1's (since $\bar{n}$ people are getting the higher quality good), and the rest can have any two possible outcomes, $\varepsilon=0,-1$, that is they may have got the lower quality good, or may not have got any good at all. Hence we get the following:

$$
\sum_{\substack{\bar{v} \in \Omega \\
\varepsilon_{i}(\bar{v})=0}} 1=\left(\begin{array}{c}
n-1 \\
\bar{n}
\end{array}\right) \times 2^{(n-1-\bar{n})} .
$$


Similarly, $\left(\sum_{\substack{\bar{v} \in \Omega \\ \varepsilon_{i}(\bar{v})=1}} 1\right)$ counts the number of vectors of length $(n-1)$ with $(\bar{n}-1)$ number of 1's (since the $i$ th person has got the higher quality good), and is given by

$$
\sum_{\substack{\bar{v} \in \Omega \\
\varepsilon_{i}(\bar{v})=1}} 1=\left(\begin{array}{c}
n-1 \\
\bar{n}-1
\end{array}\right) \times 2^{(n-1)-(\bar{n}-1)}=\left(\begin{array}{c}
n-1 \\
\bar{n}-1
\end{array}\right) \times 2^{(n-\bar{n})} .
$$

Substituting these in the above expression, and letting $\sum_{i} e_{i l}=e_{L}$ and $\sum_{i} e_{i h}=$ $e_{H}$, we can solve $\mathcal{K}$ as following:

$$
\mathcal{K}=\frac{1}{\left(\begin{array}{l}
n \\
\bar{n}
\end{array}\right) 2^{(n-\bar{n}-1)}\left\{e_{L}\left(\frac{n-\bar{n}}{n}\right)+2 \frac{\bar{n}}{n} e_{H}\right\}} .
$$




\section{Appendix (Not Necessary For Online Publication)}

\section{Appendix G. Correlation}

Let us see the correlation between the outcomes of two individuals, that is, say we are interested in the correlation of the outcomes players 1 and 2, $\operatorname{Corr}\left(\varepsilon_{1}, \varepsilon_{2}\right)$. Now

$$
\begin{aligned}
\operatorname{Corr}\left(\varepsilon_{1}, \varepsilon_{2}\right) & =\frac{\operatorname{Cov}\left(\varepsilon_{1}, \varepsilon_{2}\right)}{\sqrt{\operatorname{Var}\left(\varepsilon_{1}\right) \operatorname{Var}\left(\varepsilon_{2}\right)}} \\
& =E\left(\varepsilon_{1} \varepsilon_{2}\right)-E\left(\varepsilon_{1}\right) E\left(\varepsilon_{2}\right) .
\end{aligned}
$$

We know (from calculations before) that $E\left(\varepsilon_{1}\right)=\operatorname{Pr}\left\{\varepsilon_{1}\right\}=1$. Similarly $E\left(\varepsilon_{2}\right)=\operatorname{Pr}\left\{\varepsilon_{2}=1\right\}$. Also $E\left(\varepsilon_{1} \varepsilon_{2}\right)=\operatorname{Pr}\left\{\varepsilon_{1} \varepsilon_{2}\right\}=1$ (since in all other possibilities, $\varepsilon_{1}$ or $\varepsilon_{2}$ or both are 0 and there is no contribution to the expectation). Now (restricting to the $\lambda=0$ case), we have

$$
\begin{aligned}
\operatorname{Pr}\left\{\varepsilon_{1}=1, \varepsilon_{2}=1\right\} & =\sum_{\substack{\mathbf{v} \in \Omega \\
\varepsilon_{1}(\mathbf{v})=1 \\
\varepsilon_{2}(\mathbf{v})=1}} p_{\mathbf{v}} \\
& =\sum_{\substack{\mathbf{v} \in \Omega \\
\varepsilon_{1}(\mathbf{v})=1 \\
\varepsilon_{2}(\mathbf{v})=1}} K\left[\sum_{i=1}^{n} \varepsilon_{i}(\mathbf{v}) e_{i}\right]
\end{aligned}
$$

(where $K$ is as given in (4) with $\lambda=0$ )

$$
\begin{aligned}
& =K\left[\sum_{i=1}^{n} e_{i} \sum_{\substack{\mathbf{v} \in \Omega \\
\varepsilon_{1}(\mathbf{v})=1 \\
\varepsilon_{2}(\mathbf{v})=1}} \varepsilon_{i}(\mathbf{v})\right] \\
& =K\left[\sum_{i=1}^{n} e_{i} \sum_{\substack{\mathbf{v} \in \Omega \\
\varepsilon_{1}(\mathbf{v})=1 \\
\varepsilon_{2}(\mathbf{v})=1 \\
\varepsilon_{i}(\mathbf{v})=1}} 1\right] .
\end{aligned}
$$


Now

$$
\sum_{\substack{\mathbf{v} \in \Omega \\
\varepsilon_{1}(\mathbf{v})=1 \\
\varepsilon_{2}(\mathbf{v})=1 \\
\varepsilon_{i}(\mathbf{v})=1}} 1=\left\{\begin{array}{lll}
\left(\begin{array}{l}
n-2 \\
\bar{n}-2
\end{array}\right) & \text { if } & i=1 \\
\left(\begin{array}{ll}
n-2 \\
\bar{n}-2
\end{array}\right) & \text { if } & i=2 \\
\left(\begin{array}{l}
n-3 \\
\bar{n}-3
\end{array}\right) & \text { if } & i \neq 1,2
\end{array} .\right.
$$

Substituting and simplifying, we get

$$
\operatorname{Pr}\left\{\varepsilon_{1}=1, \varepsilon_{2}=1\right\}=\frac{(\bar{n}-1)(\bar{n}-2)}{(n-1)(n-2)}+\frac{\left(e_{1}+e_{2}\right)}{\sum_{i} e_{i}} \frac{(n-\bar{n})(\bar{n}-1)}{(n-1)(n-2)} .
$$

Recall that

$$
\operatorname{Pr}\left\{\varepsilon_{i}=1\right\}=\frac{\bar{n}-1}{n-1}+\frac{e_{i}}{\sum_{i} e_{i}} \frac{n-\bar{n}}{n-1} .
$$

Hence we get

$$
\begin{aligned}
\operatorname{Cov}\left(\varepsilon_{1}, \varepsilon_{2}\right)= & \frac{(\bar{n}-1)(\bar{n}-2)}{(n-1)(n-2)}+\frac{\left(e_{1}+e_{2}\right)}{\sum_{i} e_{i}} \frac{(n-\bar{n})(\bar{n}-1)}{(n-1)(n-2)} \\
& -\left[\frac{\bar{n}-1}{n-1}+\frac{e_{1}}{\sum_{i} e_{i}} \frac{n-\bar{n}}{n-1}\right]\left[\frac{\bar{n}-1}{n-1}+\frac{e_{2}}{\sum_{i} e_{i}} \frac{n-\bar{n}}{n-1}\right] .
\end{aligned}
$$

Also we can calculate that

$$
\operatorname{Var}\left(\varepsilon_{1}\right)=\left(\operatorname{Pr}\left\{\varepsilon_{1}=1\right\}\right)\left(1-\operatorname{Pr}\left\{\varepsilon_{1}=1\right\}\right) .
$$

Now by substituting all the expressions we can get $\operatorname{Corr}\left(\varepsilon_{1}, \varepsilon_{2}\right)$. Now, in order to keep the calculations tractable, we make the simplifying assumption of letting $n$ being large (so that $\frac{1}{n} \approx 0$ ). In this case, dividing the expression for $\operatorname{Cov}\left(\varepsilon_{1}, \varepsilon_{2}\right)$ throughout by $n$ and letting $\frac{1}{n} \approx 0$, we get

$$
\begin{aligned}
\operatorname{Cov}\left(\varepsilon_{1}, \varepsilon_{2}\right)= & \frac{\bar{n}^{2}}{n}+\frac{\left(e_{1}+e_{2}\right)}{\sum_{i} e_{i}} \frac{\bar{n}}{n}\left(1-\frac{\bar{n}}{n}\right) \\
& -\left[\frac{\bar{n}}{n}+\frac{e_{1}}{\sum_{i} e_{i}}\left(1-\frac{\bar{n}}{n}\right)\right]\left[\frac{\bar{n}}{n}+\frac{e_{2}}{\sum_{i} e_{i}}\left(1-\frac{\bar{n}}{n}\right)\right] .
\end{aligned}
$$

Simplifying the above expression we get

$$
\operatorname{Cov}\left(\varepsilon_{1}, \varepsilon_{2}\right)=-\frac{\left(e_{1} e_{2}\right)}{\left(\sum_{i} e_{i}\right)^{2}}\left(1-\frac{\bar{n}}{n}\right)^{2}<0
$$

Hence $\operatorname{Corr}\left(\varepsilon_{1}, \varepsilon_{2}\right)<0$. In fact, we can calculate the expression for $\operatorname{Corr}\left(\varepsilon_{1}, \varepsilon_{2}\right)$ more precisely in this case. Notice that when $n$ is large

$$
\operatorname{Pr}\left\{\varepsilon_{1}=1\right\}=\frac{\bar{n}}{n}+\frac{e_{1}}{\sum_{i} e_{i}}\left(1-\frac{\bar{n}}{n}\right) .
$$


And variance can be calculated as

$$
\operatorname{Var}\left(\varepsilon_{1}\right)=\left(\frac{\bar{n}}{n}+\frac{e_{1}}{\sum_{i} e_{i}}\left(1-\frac{\bar{n}}{n}\right)\right)\left(1-\frac{\bar{n}}{n}\right)\left(1-\frac{e_{1}}{\sum_{i} e_{i}}\right) .
$$

Hence substituting and simplifying, we get the expression for the correlation coefficient as below:

$$
\begin{aligned}
\operatorname{Corr}\left(\varepsilon_{1}, \varepsilon_{2}\right) & =-\frac{\frac{e_{1} e_{2}}{\left(\sum_{i} e_{i}\right)^{2}}}{\sqrt{\left(\frac{\bar{n}}{n}+\frac{e_{1}}{\sum_{i} e_{i}}\left(1-\frac{\bar{n}}{n}\right)\right)\left(1-\frac{e_{1}}{\sum_{i} e_{i}}\right)\left(\frac{\bar{n}}{n}+\frac{e_{2}}{\sum_{i} e_{i}}\left(1-\frac{\bar{n}}{n}\right)\right)\left(1-\frac{e_{2}}{\sum_{i} e_{i}}\right)}} \\
& <0 .
\end{aligned}
$$

Notice that the correlation coefficient is generalizable to that between outcomes of any two individuals $i$ and $j$ after appropriately substituting for 1 and 2 in the expression on the R.H.S. Hence we see that, as expected, the correlation between outcomes of any two individuals is negative, that is, as one person's chance of getting a better-quality good increases, that of the other falls. The following proposition summarizes the findings.

Proposition 12. The correlation coefficient between the outcomes of any two individuals, say individual $i$ and individual $j, i \neq j$, is given as

$$
\begin{aligned}
\operatorname{Corr}\left(\varepsilon_{i}, \varepsilon_{j}\right) & =-\frac{\frac{e_{i} e_{j}}{\left(\sum_{i} e_{i}\right)^{2}}}{\sqrt{\left(\frac{\bar{n}}{n}+\frac{e_{i}}{\sum_{i} e_{i}}\left(1-\frac{\bar{n}}{n}\right)\right)\left(1-\frac{e_{i}}{\sum_{i} e_{i}}\right)\left(\frac{\bar{n}}{n}+\frac{e_{j}}{\sum_{i} e_{i}}\left(1-\frac{\bar{n}}{n}\right)\right)\left(1-\frac{e_{j}}{\sum_{i} e_{i}}\right)}} \\
& =\frac{-\frac{e_{i} e_{j}}{\bar{e}^{2}}}{\sqrt{\left(\bar{n}+\frac{e_{i}}{\bar{e}}\left(1-\frac{\bar{n}}{n}\right)\right)\left(n-\frac{e_{i}}{\bar{e}}\right)\left(\bar{n}+\frac{e_{j}}{\bar{e}}\left(1-\frac{\bar{n}}{n}\right)\right)\left(n-\frac{e_{j}}{\bar{e}}\right)}} \\
& <0 .
\end{aligned}
$$

Moreover, in case the number of better quality goods is small relative to the total number of consumers, so that $\frac{\bar{n}}{n} \approx 0$, we can further simplify the expression of correlation coefficient to arrive at the following corollary.

Corollary 2. If $\frac{\bar{n}}{n} \approx 0$ then we get

$$
\operatorname{Corr}\left(\varepsilon_{i}, \varepsilon_{j}\right)=-\sqrt{\frac{e_{i} e_{j}}{\left(\sum_{i} e_{i}-e_{i}\right)\left(\sum_{i} e_{i}-e_{j}\right)}} .
$$

\section{Appendix H. LOTTERY}

Consider a situation in which each person can buy more than one ticket for a limited number of prizes. Say there are $k$ number of people, $n$ the total number 
of tickets for $\bar{n}$ number of prizes (seats), with $n>\bar{n}$. Let $e_{i}$ be the number of tickets purchased by player $i$ (note this has nothing to do with effort as of now). Assume all available tickets are bought, that is, $\sum_{i=1}^{k} e_{i}=n$ and also $e_{i} \in\{1,2, \ldots, n-k+1\}$. Hence the space of all outcomes $\Omega$, is as follows:

$$
\begin{aligned}
\Omega:= & \left\{\mathbf{v}=\left(\varepsilon_{11}(\mathbf{v}), \ldots, \varepsilon_{1 e_{1}}(\mathbf{v}), \varepsilon_{21}(\mathbf{v}), \ldots, \varepsilon_{2 e_{2}}(\mathbf{v}), \ldots, \varepsilon_{k 1}(\mathbf{v}), \ldots, \varepsilon_{k e_{k}}(\mathbf{v})\right)\right. \\
& \left.\in\{0,1\}^{n}: \sum_{i=1}^{k} \sum_{j=1}^{e_{i}} \varepsilon_{i j}(\mathbf{v})=\bar{n}\right\}
\end{aligned}
$$

where $\varepsilon_{k e_{k}}(\mathbf{v})$ is the $n$-th component of the vector $\mathbf{v}$.

The restriction $\sum_{i=1}^{k} \sum_{j=1}^{e_{i}} \varepsilon_{i j}(\mathbf{v})=\bar{n}$ reflects the fact that in any outcome all the prizes are won by someone. Moreover the first $e_{1}$ outcomes in $\mathbf{v}$ reflects the outcomes for the tickets bought by player 1 . Note that here $\varepsilon_{i j}(\mathbf{v})=1$ means that in the outcome $\mathbf{v}$ the $i$-th person has won in the $j$ th ticket and otherwise $\varepsilon_{i j}(\mathbf{v})=0$. Also note that the number of outcomes in the space is given by

$$
|\Omega|=\left(\begin{array}{l}
n \\
\bar{n}
\end{array}\right)=\frac{n !}{\bar{n} !(n-\bar{n}) !} .
$$

Now let us compute the probability that any one person, say person 1 , has won $p$ prizes (where $p \leq e_{1}$, that is number of prizes won is less than the number of tickets bought by person 1 and $p \leq \bar{n}$, that is the number of prizes won is less than the total number of prizes). Now all outcomes are equally likely. Moreover person 1 wins p prizes with $e_{1}$ number of tickets in $\left(\begin{array}{c}e_{1} \\ p\end{array}\right)$ ways. For each such way, the rest $\bar{n}-p$ prizes can be won by $n-e_{1}$ tickets in $\left(\begin{array}{c}n-e_{1} \\ \bar{n}-p\end{array}\right)$. Hence the probability is given by:

$$
\begin{aligned}
\operatorname{Pr}\{\text { Player } 1 \text { wins } p \text { prizes }\}= & \sum_{\substack{\mathbf{v} \in \Omega \\
\varepsilon_{11}(\mathbf{v})+\cdots+\varepsilon_{1 e_{1}}(\mathbf{v})=p}} \frac{1}{\left(\begin{array}{l}
n \\
\bar{n}
\end{array}\right)} \\
& =\frac{\left(\begin{array}{c}
e_{1} \\
p
\end{array}\right)\left(\begin{array}{c}
n-e_{1} \\
\bar{n}-p
\end{array}\right)}{\left(\begin{array}{c}
n \\
\bar{n}
\end{array}\right)} .
\end{aligned}
$$

More generally, the probability that the $\ell$ th player will win $p$ prizes is given by:

$$
\text { Pr }\{\text { Player } \ell \text { wins } p \text { prizes }\}=\frac{\left(\begin{array}{c}
e_{l} \\
p
\end{array}\right)\left(\begin{array}{l}
n-e_{l} \\
\bar{n}-p
\end{array}\right)}{\left(\begin{array}{l}
n \\
\bar{n}
\end{array}\right)}
$$


Now our model can be related to this lottery set-up in the following way: Let each ticket, for example, to be a unit of effort exerted, so that more number of tickets will correspond to more effort exerted (of course, effort in our model is a continuous variable while number of tickets can only be discrete). If tickets have a (uniform) price, that might be interpreted as $\chi$ in our model. Hence the total expenditure, of person $i$, to purchase $e_{i}$ number of tickets, would be $\chi e_{i}$, the total 'effort cost' of player $i$ in our model (assuming $\alpha=0$, or linear cost of effort).

Interestingly, in the (usual) case with every person buying only one ticket $e_{i}=1, \forall i$ (or alternatively the symmetric effort case with everybody exerting the same effort), the probability that the $i$ th person wins is given by

$$
\operatorname{Pr}\{\text { Player } i \text { wins } p \text { prizes }\}=\frac{\left(\begin{array}{l}
1 \\
1
\end{array}\right)\left(\begin{array}{l}
n-1 \\
\bar{n}-1
\end{array}\right)}{\left(\begin{array}{l}
n \\
\bar{n}
\end{array}\right)}=\frac{\bar{n}}{n} .
$$

which is the usual random probability model that we had.

Ecole Normale Supérieure de Cachan and CES (Centre D'économie de la Sorbonne)

E-mail address: andre.depalma@ens-cachan.fr

Indian Institute of Management Bangalore (IIMB)

E-mail address: soumyanetra.munshi@iimb.ernet.in 